\title{
Almost slender rings and compact rings
}

Jensen, Chr Ulrik; Jøndrup, Søren; Thorup, Anders

Published in:

Journal of Pure and Applied Algebra

DOI:

10.1016/j.jpaa.2018.08.005

Publication date:

2019

Document version

Early version, also known as pre-print

Citation for published version (APA):

Jensen, C. U., Jøndrup, S., \& Thorup, A. (2019). Almost slender rings and compact rings. Journal of Pure and Applied Algebra, 223(5), 1869-1896. https://doi.org/10.1016/j.jpaa.2018.08.005 


\title{
Almost slender rings and compact rings
}

\author{
Christian U. Jensen, Søren Jøndrup and Anders Thorup \\ Department of Mathematical Sciences, University of Copenhagen
}

Revised version 2 May 2018

\begin{abstract}
Classical results concerning slenderness for commutative integral domains are generalized to commutative rings with zero divisors. This is done by extending the methods from the domain case and bringing them in connection with results on the linear topologies associated to non-discrete Hausdorff filtrations. In many cases a weakened notion "almost slenderness" of slenderness is appropriate for rings with zero divisors. Special results for countable rings are extended to rings said to be of "bounded type" (including countable rings, 'small' rings, and, for instance, rings that are countably generated as algebras over an Artinian ring).

More precisely, for a ring $R$ of bounded type it is proved that $R$ is slender if $R$ is reduced and has no simple ideals, or if $R$ is Noetherian and has no simple ideals; moreover, $R$ is almost slender if $R$ is not perfect (in the sense of H. Bass). We use our methods to study various special classes of rings, for instance von Neumann regular rings and valuation rings. Among other results we show that the following two rings are slender: the ring of Puiseux series over a field and the von Neumann regular ring $k^{\mathbb{N}} / k^{(\mathbb{N})}$ over a von Neumann regular ring $k$.

For a Noetherian ring $R$ we prove that $R$ is a finite product of local complete rings iff $R$ satisfies one of several (equivalent) conditions of algebraic compactness. A 1-dimensional Noetherian ring is outside this 'compact' class precisely when it is almost slender. For the rings of classical algebraic geometry we prove that a localization of an algebra finitely generated over a field is either Artinian or almost slender. Finally, we show that a Noetherian ring $R$ is a finite product of local complete rings with finite residue fields exactly when there exists a map of $R$-algebras $R^{\mathbb{N}} \rightarrow R$ vanishing on $R^{(\mathbb{N})}$.
\end{abstract}

\section{Table of contents}

1. Introduction and setup.

2. General rings.

3. "Countability" conditions.

4. Von Neumann regular rings and valuation rings.

5. Examples and minor results.

6. Noetherian rings.

7. Augmenting the product algebra.

2010 Mathematics Subject Classification. 13A18, 13J10, 13C99; secondary 12L10, 13F10, 20 K25.

Key words and phrases. slender ring, algebraically compact ring, Noetherian ring, Artinian ring, valuation ring, von Neumann regular ring, graded ring. 


\section{Introduction and setup}

The notion slenderness was first introduced by J. Loś in 1954 for abelian groups and has since then be generalized in various directions. Slenderness and compactness are in some sense extreme opposites. In the previous paper [JJT] we elaborated on this point for commutative integral domains, where unexpected connections turned up. In the present paper we extend the work to commutative rings with zero divisors, where several new obstacles appear. New proof ideas - lying in the intersection of set theory, topology and ring theory - are needed; some of them may be of independent interest.

The admission of zero divisors for the rings is in some sense parallel to the admission of torsion elements in modules over domains. For the module case there is a well-known natural weakening of slenderness, called almost slenderness, see [E-M, p. 57].

For rings with zero divisors the notion of being slender, transferred verbatim from domains, is not adequate in all cases, and it is natural to consider in addition a similar weakening, the notion of being almost slender.

After an introduction of the basic concepts and definitions the next Section 2 gives a number of results concerning general (commutative) rings. We recall the fundamental result of Dimitric that a ring is slender iff it is halfslender and not complete with respect to any linear topology defined by a (countable) non-discrete Hausdorff filtration of ideals. Many additional results in Section 2 are based on a technical key lemma 2.6. They concern the question of slenderness/almost slenderness with respect to ring extensions $R_{0} \subseteq R$. For instance, we analyze in detail conditions under which graded rings and their subrings are slender/almost slender, see 2.7 and 2.8. A similar analysis of conditions for the slenderness of a "localization" (ring of fractions) $R \subseteq R\left[S^{-1}\right]$ is much more delicate, see 2.9. The final result of the Section, Proposition 2.11 describes various filtrations with respect to which a nonslender ring is complete.

The following Section 3 deals with rings that roughly speaking are not "too large". There are several results on 'small' rings, that is, rings of cardinality strictly less than $2^{\aleph_{0}}$, see for instance [EB-K1], where modules over not necessarily commutative rings are considered. We generalize this notion to rings of bounded type, which are rings that in some sense are 'small' as algebras over a field, see Definition 3.1, and we prove results similar to those obtained in [EB-K1]. In fact, we extend some of their results to the non-reduced case (Theorem 3.12), and we present a non-trivial generalization to rings that are 'of bounded type' as algebras over an Artinian ring.

Sections 4 and 5 contain a series of rather concrete applications to well-known classes of rings, for instance von Neumann regular rings, valuation rings and perfect rings.

Section 6 contains a surprisingly simple characterization: For Noetherian rings, the classes of algebraically compact, $\aleph_{0}$-compact, and nearly compact rings are identical, and equal to the class of semilocal complete rings; moreover, a ring in this class is complete with respect to the linear topology defined by any non-discrete Hausdorff filtration. For 1-dimensional Noetherian rings it is proved that this class of compact rings is exactly the complement to the class of almost slender rings, see Theorem 6.6. The slender Noetherian rings were characterized by El Bashir and Kepka [EB-K2, Corollary 4.4, p. 2590]. Their main result is that a nonslender ring without simple ideals is complete with respect to a linear topology defined by some non-discrete Hausdorff filtration. We recover their result, and prove in the reduced case that the ideals of the filtration may be taken to be principal ideals, see Corollary 6.11.

In the terminology below, a ring is not halfslender if there exists a non-zero $R$-linear map $R^{\mathbb{N}} / R^{(\mathbb{N})} \rightarrow R$. The last Section 7 characterizes Noetherian rings $R$ for which there 
exists such a map which in addition is a map of rings.

Setup 1.1. We first fix some notations. All rings will be commutative and unital, and ring homomorphisms carry 1 to 1 . We fix a commutative ring $R$, assumed to be non-zero so that $1 \neq 0$. The invertible elements of $R$ will be called units, and non-zero divisors are called regular elements. We use the notation $R\left[S^{-1}\right]$ for the ring of fractions formed with respect to a given subset $S \subset R$; its elements are formal fractions $a / s$ where $a \in R$ and $s$ is a finite product of elements of $S$. We denote by $\mathbb{N}$ the set of non-negative integers $0,1,2, \ldots$ The product $R^{\mathbb{N}}$ is an $R$-module, but occasionally we use the coordinatewise multiplication in $R^{\mathbb{N}}$. For any subset $J \subseteq \mathbb{N}$ we denote by $1_{J}$ the characteristic function of $J$ viewed as a sequence in $R^{\mathbb{N}}$, and $R^{J}$ is viewed as the submodule of $R^{\mathbb{N}}$ consisting of sequences $x$ with $x_{i}=0$ for $i \notin J$. In particular, for $a \in R$ the product $a 1_{\mathbb{N}}$ is the constant sequence with value $a$, and the product $x 1_{J}$, for a sequence $x \in R^{\mathbb{N}}$, is the sequence whose $j$ 'th coordinate is $x_{j}$ for $j \in J$ and zero otherwise. Note that $1_{n}$ is the characteristic function of $\{n\}$.

A (countable) non-discrete Hausdorff filtration in $R$ is a sequence of ideals,

$$
R \supseteq \mathfrak{a}_{1} \supseteq \mathfrak{a}_{2} \supseteq \cdots, \text { with } \mathfrak{a}_{n} \neq 0 \text { for all } n \text {, and } \bigcap \mathfrak{a}_{n}=0 \text {. }
$$

The filtration defines a linear topology on $R$ is which the $\mathfrak{a}_{n}$ form a basis for the neighborhoods of 0 . The ring is complete with respect to the filtration if every Cauchy sequence is convergent, or, equivalently, if the canonical inclusion of $R$ into the completion $\widehat{R}:=\lim R / \mathfrak{a}_{n}$ is an isomorphism. For a Noetherian local or semilocal ring complete without further specification is always with respect to the filtration of powers of the Jacobson ideal.

The concepts extend verbatim to filtrations by submodules in an $R$-modules.

Definition 1.2. For an $R$-linear map $\varphi: R^{\mathbb{N}} \rightarrow R$, the value $\varphi_{n}:=\varphi\left(1_{n}\right)$ is its $n$ 'th coordinate. Note that the assignment $\varphi \rightarrow\left(\varphi_{n}\right)$, from the map to its sequence of coordinates, is given by the linear restriction map,

$$
\operatorname{Hom}_{R}\left(R^{\mathbb{N}}, R\right) \rightarrow \operatorname{Hom}_{R}\left(R^{(\mathbb{N})}, R\right)=R^{\mathbb{N}}
$$

In general, the map $\left(^{*}\right)$ is neither surjective, nor injective.

Our work is centered about the relations between four properties of the ring $R$, some of which are most conveniently defined through their negations:

(sl) $R$ is slender if for every linear map $\varphi: R^{\mathbb{N}} \rightarrow R$ there is only a finite number of $n$ such that $\varphi_{n} \neq 0$.

(as) $R$ is almost slender if for every linear map $\varphi: R^{\mathbb{N}} \rightarrow R$ there is only a finite number of $n$ such that $\varphi_{n}$ is regular in $R$.

(not nl-c) $R$ is not nearly compact if for every linear map $\varphi: R^{\mathbb{N}} \rightarrow R$ and every regular element $u$ there is only a finite number of $n$ such that $\varphi_{n}=u$.

(not $\aleph_{0}$-c) $R$ is not $\aleph_{0}$-compact if for every linear map $\varphi: R^{\mathbb{N}} \rightarrow R$ there is only a finite number of $n$ such that $\varphi_{n}=1$, or, equivalently, such that $\varphi_{n}$ is invertible.

It is easy to obtain the following characterizations (see [E-M, chap. 3]):

(not sl) $R$ is nonslender iff there exists a linear map $\varphi: R^{\mathbb{N}} \rightarrow R$ such that $\varphi_{n} \neq 0$ for all $n$. (not as) $R$ is not almost slender iff there exists a linear map $\varphi: R^{\mathbb{N}} \rightarrow R$ such that $\varphi_{n}$ is regular for all $n$.

(nl-c) $R$ is nearly compact iff there exist a regular element $u$ and a linear map $\varphi: R^{\mathbb{N}} \rightarrow R$ such that $\varphi_{n}=u$ for all $n$. 
$\left(\aleph_{0}\right.$-c) $R$ is $\aleph_{0}$-compact iff there exists a linear map $\varphi: R^{\mathbb{N}} \rightarrow R$ such that $\varphi_{n}=1$ for all $n$. Clearly, if such a map exists then there exists a linear map $\psi: R^{\mathbb{N}} \rightarrow R$ such that the sequence $\psi_{n}$ has prescribed values. In other words, $R$ is $\aleph_{0}$-compact iff the restriction map $(*)$ is surjective.

Let us note in passing that the ring $R$ is called algebraically compact or pureinjective if the maps obtained from $(*)$ by replacing $\mathbb{N}$ by arbitrary sets $I$ are surjective, see [J-L, p. 126].

In addition, by composing $\varphi$ with the automorphism $\alpha(x)=\left(x_{0}, x_{1}-x_{0}, x_{2}-x_{1}, \ldots\right)$ of $R^{\mathbb{N}}$ and its inverse (see [JJT, Remark 1.3, p. 895]), we obtain for any fixed $u \in R$ : There exists a linear map $\varphi: R^{\mathbb{N}} \rightarrow$ with $\varphi_{n}=u$ for all $n$ iff there exists a linear map $\psi: R^{\mathbb{N}} \rightarrow R$ with $\psi_{n}=0$ for all $n$ and $\psi\left(1_{\mathbb{N}}\right)=u$. As a consequence, two of the characterizations above can be reformulated:

(nl-c') $R$ is nearly compact iff there exists a linear map $\varphi: R^{\mathbb{N}} \rightarrow R$ such that $\varphi_{n}=0$ for all $n$ and $\varphi\left(1_{\mathbb{N}}\right)$ is a regular element.

$\left(\aleph_{0}\right.$-c') $R$ is $\aleph_{0}$-compact iff there exists a linear map $\varphi: R^{\mathbb{N}} \rightarrow R$ such that $\varphi_{n}=0$ for all $n$ and $\varphi\left(1_{\mathbb{N}}\right)=1$.

In addition, it follows that $\operatorname{Hom}\left(R^{\mathbb{N}} / R^{(\mathbb{N})}, R\right)=0$ iff there is no linear map $\varphi: R^{\mathbb{N}} \rightarrow R$ such that $\varphi_{n}=u$ is a non-zero constant for all $n$. A ring $R$ with this property is called halfslender, see [EB-K1, p. 1576]. As $\operatorname{Hom}\left(R^{\mathbb{N}} / R^{(\mathbb{N})}, R\right)$ is the kernel of the map (*), it follows that $R$ is halfslender iff the map (*) is injective. Note finally that an integral domain $R$ is halfslender iff it is not nearly compact.

Note 1.3. Clearly (sl) $\Rightarrow$ (as) $\Rightarrow$ (not nl-c) $\Rightarrow$ (not $\aleph_{0}$-c). Moreover, (sl) $\Leftrightarrow$ (as) for a domain, and (as) $\Leftrightarrow$ (not nl-c) $\Leftrightarrow\left(\right.$ not $\left.\aleph_{0}-\mathrm{c}\right)$ for a ring in which every regular element is invertible.

None of the three implications is, in general, reversible. For the first implication, (sl) $\Rightarrow$ (as), a simple counterexample for the reverse is the ring $R=\mathbb{Q} \times \mathbb{Z}$ which is almost slender and nonslender; see also Examples 5.3 and 5.4 for irreducible rings. For the second implication, (as) $\Rightarrow$ (not nl-c), a local Noetherian counterexample for the reverse is the ring $\mathbb{Z}_{(p)}[[X]]$ which is not nearly compact and not almost slender, see Example 5.1. The last implication, (not nl-c) $\Rightarrow$ (not $\left.\aleph_{0}-\mathrm{c}\right)$, or $\left(\aleph_{0}-\mathrm{c}\right) \Rightarrow(\mathrm{nl}-\mathrm{c})$, is an equivalence for Noetherian rings, see Theorem 6.3. In the non Noetherian case a counterexample to the reverse implication is given by the subring of power series in $\mathbb{Q}[[X]]$ whose constant term belongs to $\mathbb{Z}_{(p)}$, see Example 5.10. Further examples are collected in Section 5.

\section{General rings}

In this section we recall and comment on the result of Dimitric on the connection between slenderness and completeness with respect to a non-discrete Haussdorff filtration. However, our main focus is on extensions of rings. For instance it is proved that "most" $\mathbb{N}$ - and $\mathbb{Z}$ graded rings are slender (Propositions 2.7 and 2.8). In addition we consider a localization $R\left[S^{-1}\right]$, countably generated over $R$, and we provide general criteria under which the localization is slender (Proposition 2.9). Our results imply for instance for a Noetherian domain $R$, not a field, that the ring of formal Laurent series $R[[X]]\left[X^{-1}\right]$ is slender, see Note 2.10. The question of slenderness for arbitrary localizations $R \subset R\left[S^{-1}\right]$ is quite subtle, and we have only partial results. The case when $R$ is a finitely generated algebra over a field is solved in Section 6 (Theorem 6.4). For a Noetherian domain $R$ it is known that a proper localization is $\aleph_{0}$-compact iff the localization is the field of fractions of $R$, see [JJT, Theorem 4.2, p. 901], but we have no similar results for general Noetherian rings. 
In the last parts of the section we consider various non-discrete Hausdorff filtrations with respect to which a nonslender ring is complete.

Lemma 2.1. A field is nonslender. A ring containing a simple ideal is nonslender.

Proof. As is well known it follows by simple vector space theory that a field is even $\aleph_{0^{-}}$ compact, and in particular nonslender.

A simple ideal (that is, simple as an $R$-module) in $R$ defines an $R$-linear embedding $K:=R / \mathfrak{m} \hookrightarrow R$ where $\mathfrak{m}$ is the annihilator of a non-zero element $u$ in the simple ideal. Now $K$ as a residue ring of $R$ is a field, and hence $\aleph_{0}$-compact. Let $\tilde{\varphi}: K^{\mathbb{N}} \rightarrow K$ be a $K$-linear map such that $\tilde{\varphi}_{n}=1$ for all $n$. Then $\tilde{\varphi}$ is also $R$-linear, and for the $R$-linear composition,

$$
\varphi: R^{\mathbb{N}} \rightarrow K^{\mathbb{N}} \rightarrow K \hookrightarrow R
$$

we have $\varphi_{n}=u \neq 0$ for all $n$. Hence $R$ is nonslender (in fact, not even halfslender).

Lemma 2.2. The ring $R$ is nonslender iff there exists an $R$-linear map $\varphi: R^{\mathbb{N}} \rightarrow R$ such that all the ideals $\varphi\left(R^{[n, \infty)}\right)$ are non-zero. Moreover, if $\varphi$ is such a map and $u_{n} \in \varphi\left(R^{[n, \infty)}\right)$ for $n=0,1,2, \ldots$ is any sequence of non-zero elements then there exists an $R$-linear map $\psi: R^{\mathbb{N}} \rightarrow R$ such that $\psi_{n}=u_{n}$ and $\psi\left(R^{[n, \infty)}\right) \subseteq \varphi\left(R^{[n, \infty)}\right)$ for all $n$.

Proof. "Only if" is obvious. To prove the "if" part, let $\varphi$ be such a map, and choose non-zero elements $u_{n} \in \varphi\left(R^{[n, \infty)}\right)$ for $n=0,1,2, \ldots$ Pick for every $n$ a sequence $v^{(n)} \in R^{[n, \infty)}$ such that $\varphi\left(v^{(n)}\right)=u_{n}$. Then there is a well-defined $R$-linear map $\psi: R^{\mathbb{N}} \rightarrow R$, given by

$$
\psi(x)=\varphi(\alpha(x)), \quad \text { where } \alpha(x):=\sum x_{i} v^{(i)} .
$$

Obviously $\psi_{n}=u_{n}$, and $\alpha\left(R^{[n, \infty)}\right) \subseteq R^{[n, \infty)}$. The existence of $\psi$ is a consequence. The existence implies in particular that $R$ is nonslender.

Remark 2.3. Assume that $R$ is nonslender, so that there exists an $R$-linear $\varphi$ as in Lemma 2.2. Set $\mathfrak{a}_{n}:=\varphi\left(R^{[n, \infty)}\right)$ and $\mathfrak{a}:=\bigcap \mathfrak{a}_{n}$. If the inclusion $\mathfrak{a} \subseteq \mathfrak{a}_{n}$ is strict for all $n$, or, equivalently, if the inclusion $\mathfrak{a}_{n+1} \subseteq \mathfrak{a}_{n}$ is strict for infinitely many $n$, then the $\mathfrak{a}_{n} / \mathfrak{a}$ form a non-discrete Hausdorff filtration of the residue ring $R / \mathfrak{a}$, and, by a well-known argument of Dimitrić, [D, 2.2 Lemma, p. 378], $R / \mathfrak{a}$ is complete with respect to this filtration. In particular, if $\mathfrak{a}=0$, then $R$ is complete with respect to the filtration $\left(\mathfrak{a}_{n}\right)$.

If $\mathfrak{a} \neq 0$, take a non-zero element $u \in \mathfrak{a}$. It follows from Lemma 2.2 that there exists an $R$-linear map $\psi: R^{\mathbb{N}} \rightarrow R$ with $\psi_{n}=u$ for all $n$. Consequently, as noted in Definition $1.2, R$ is not halfslender.

Note that these observations are the essentials for the "if" part in the following fundamental result of Dimitrić [D, 4.4. Corollary, p. 382]: $R$ is slender iff $R$ is halfslender and not complete with respect to any non-discrete Hausdorff filtration.

The "only if" part is the first assertion of Proposition 2.11 below.

Proposition 2.4. Assume that a subring $R_{0} \subseteq R$ is a domain, and that $R$ is finitely generated and torsion free as $R_{0}$-module. Then the following equivalences hold:

(a) $R_{0}$ is slender $\Leftrightarrow R$ is almost slender.

(b) $R_{0}$ is nearly compact $\Leftrightarrow R$ is nearly compact.

In fact, for the implication " $\Leftarrow$ " in (b) it suffices that $R_{0}$ is a domain and $R$ is torsionless as an $R_{0}$-module.

Proof. Recall that $R$ is torsionless over $R_{0}$ if for any element $u \neq 0$ in $R$ there exists an $R_{0}$-linear map $\lambda: R \rightarrow R_{0}$ such that $\lambda(u) \neq 0$. 
For the case where $R$ is a domain, a proof of the implications, and of several similar implications under various hypotheses, may be found in [JJT, Prop. 2.1, p. 897]. The proof in the case of a domain uses the fraction field $K$ of $R$ as a vector space over the fraction field $K_{0}$ of $R_{0}$. This proof may be transferred verbatim to the general case once the fraction field $K$ is replaced by the vector space $R \otimes_{R_{0}} K_{0}$ over $K_{0}$.

Proposition 2.5. Assume there is given an increasing sequence of subsets $M_{n}$ of $R$, with union $R$, and a decreasing sequence of principal ideals $q_{n} R$, and an $R$-linear map $\varphi: R^{\mathbb{N}} \rightarrow$ $R$,

$$
M_{0} \subseteq M_{1} \subseteq \cdots, \quad \bigcup_{n} M_{n}=R, \quad R=q_{0} R \supseteq q_{1} R \supseteq \cdots .
$$

Assume the following inclusions, for all $n$ :

$$
\left(M_{n}-M_{n}\right) \cap\left(q_{n} \varphi_{n}+q_{n+1} R\right) \subseteq\{0\}
$$

where the notation $M-M$ for a subset $M \subseteq R$ is used for the set of all differences $x-y$ for $x, y \in M$; in particular, $M-M=M$ for an additive subgroup $M \subseteq R$.

Then $q_{n} \varphi_{n}=0$ for $n \gg 0$.

Proof. First observe that the relation (2.5.1) for all $n$ is preserved after a restriction to an infinite subset $i_{0}<i_{1}<i_{2}<\cdots$ of indices, since $q_{i_{n+1}} R \subseteq q_{i_{n}+1} R$.

Next define the sequence $i_{0}<i_{1}<\ldots$ as follows: Set $i_{0}=0$ and choose $i_{1}>i_{0}$ such that $q_{0} \varphi_{0} \in M_{i_{1}}$. Recursively, if $i_{n-1}$ is determined choose $i_{n}>i_{n-1}$ such that $q_{i_{0}} \varphi_{i_{0}}+\cdots+q_{i_{n-1}} \varphi_{i_{n-1}} \in M_{i_{n}}$. Then, rather than restricting to the subsequence $\left(i_{n}\right)$, we may assume for all $n \geq 1$ that

$$
s_{n}:=q_{0} \varphi_{0}+\cdots+q_{n-1} \varphi_{n-1} \in M_{n} .
$$

Let $q \in R^{\mathbb{N}}$ be the sequence $q=\left(q_{0}, q_{1}, q_{2}, \ldots\right)$, and consider the value $\varphi(q)$. Then, for any $n \geq 1$ we have the equation,

$$
\varphi(q)=\varphi\left(q 1_{[0, n-1]}\right)+q_{n} \varphi_{n}+\varphi\left(q 1_{[n+1, \infty)}\right) .
$$

In the sequence $q 1_{[n+1, \infty)}$ every coordinate is a multiple of $q_{n+1}$. So, in (2.5.3), the last term belongs to $q_{n+1} R$. The first term on the right side is the sum $s_{n}$ defined in (2.5.2). Hence Equation (2.5.3) implies the relation,

$$
\varphi(q)-s_{n} \in q_{n} \varphi_{n}+q_{n+1} R
$$

By choice, $s_{n} \in M_{n}$. Choose $n \gg 0$. Then the difference in (2.5.4) belongs $M_{n}-M_{n}$. Hence, by (2.5.1), the difference equals 0 , that is, $\varphi(q)=s_{n}$. Replacing $n$ by $n+1$ we obtain $\varphi(q)=s_{n+1}$. Consequently, $s_{n+1}-s_{n}=0$. As $s_{n+1}-s_{n}=q_{n} \varphi_{n}$ we have proved that $q_{n} \varphi_{n}=0$ for $n \gg 0$.

Corollary 2.6. (Key Lemma) Assume there is given an increasing sequence of subsets $M_{n} \subseteq R$ with union $R$, and a decreasing sequence of principal ideals $q_{n} R$,

$$
M_{0} \subseteq M_{1} \subseteq \cdots, \quad \bigcup_{n} M_{n}=R, \quad R=q_{0} R \supseteq q_{1} R \supseteq q_{2} R \supseteq \cdots,
$$

satisfying the following conditions, for all $n$ :

$$
\left(M_{n}-M_{n}\right) \cap q_{n} R=\{0\} .
$$


Then:

(1) If $q_{n} \neq 0$ for all $n$, then $R$ is almost slender.

(2) If $q_{n}$ is regular for all $n$, then $R$ is slender.

Proof. Assume, indirectly, that one of the implications in (1) or (2) is false. Then there exists an $R$-linear map $\varphi: R^{\mathbb{N}} \rightarrow R$ such that $q_{n} \varphi_{n} \neq 0$ for all $n$.

Obviously, Condition (2.6.1) implies the Condition (2.5.1). Hence Proposition 2.5 implies that $q_{n} \varphi_{n}=0$ for $n \gg 0$, which is the desired contradiction.

Proposition 2.7. (Compare [E-M, III, exc. 4] or [ON, Proposition 2.9, p. 289]) Assume that $R$ embeds as a subring of an $\mathbb{N}$-graded ring $G=\bigoplus_{n \in \mathbb{N}} G_{n}$ in such a way that a non-zero element $q$ of $R$ has no constant term in $G$. Then:

(1) If $q$ is not nilpotent then $R$ is almost slender.

(2) If $q$ is a regular element of $R$ then $R$ is slender.

Moreover, if $q$ is regular in $G$ then the associated series ring $\prod_{n \in \mathbb{N}} G_{n}$ is not almost slender.

In particular, a polynomial ring in any positive number of variables over an arbitrary ring is slender, and the associated ring of formal power series is not almost slender.

Proof. For the first two assertions, let $M_{n} \subseteq R$ be the subgroup of elements of degree at most $n-1$. Since $q$ has no constant term, it follows that $q^{n}$ has no homogeneous terms of degrees less that $n$. Hence $M_{n} \cap q^{n} R=0$. Now apply Corollary 2.6 with $q_{n}:=q^{n}$.

Consider the associated series ring $\widehat{G}=\prod_{n \geq 0} G_{n}$. It may be defined as the subring of $G[[T]]$ consisting of series $\sum s_{n} T^{n}$ with $s_{n} \in G_{n}$ for all $n$. Define a $\widehat{G}$-linear map $\widehat{G}^{\mathbb{N}} \rightarrow \widehat{G}$ by the convergent series, for $x \in \widehat{G}^{\mathbb{N}}$,

$$
\varphi(x)=\sum x_{n} q^{n}
$$

Since $\varphi_{n}=q^{n}$ is regular for all $n$ it follows that $\widehat{G}$ is not almost slender.

Proposition 2.8. Assume that $R$ embeds as a subring of a $\mathbb{Z}$-graded ring $G=\bigoplus_{n \in \mathbb{Z}} G_{n}$ in such a way that a non-zero element $q \in R$ is homogeneous of positive degree in $G$. Then:

(1) If $R$ is reduced, i.e., $R$ has no non-zero nilpotents, then $R$ is almost slender.

(2) If $q$ is regular in $R$, then $R$ is slender.

Proof. For a non-zero element $s \in G$ the order and the degree, ord $s$ and deg $s$, are, respectively, the smallest and the largest degree of a non-zero homogeneous term in $s$, and the width of $s$ is the difference wd $s:=\operatorname{deg} s-\operatorname{ord} s$.

We let $M_{n} \subseteq R$ be the subgroup of elements in $R$ of degree at most $n-1$ and order at least $-n$; then any non-zero element of $M_{n}$ has width at most $2 n-1$. It suffices to prove with $p_{n}:=q\left(1+q^{n}\right)$ that any non-zero element in $p_{n} R$ has width at least $n$. Indeed, if this has been proved, then $p_{2 n} R \cap M_{n}=0$, and Corollary 2.6 applies with $q_{n}:=p_{0} \cdots p_{2 n}$.

So let $r:=s q+s q^{n+1}$ be a non-zero element of $p_{n} R$. Assume indirectly that $\mathrm{wd} r<n$. Then wd $r<\operatorname{deg} q^{n}$. Hence, by the Sublemma below applied with $s:=s q$ and $p:=-q^{n}$, we have $(s q) q^{n N}=0$ for some $N \geq 1$. In (2) $q$ is regular, and it follows that $s=0$, and hence $r=0$, a contradiction. For (1) we obtain $(s q)^{n N+1}=0$, and since $R$ has no non-zero nilpotents, it follows that $s q=0$, and hence $r=0$, a contradiction.

Sublemma. Assume in the setup of Proposition 2.8 that $p \in R$ is non-zero and homogeneous of positive degree $d$. Assume that $r:=s-p s$ is non-zero of width $\mathrm{wd} r<d$. Then there exists an integer $N \geq 1$ such that $p^{N} s=0$. 
Proof. The given equation $r=s(1-p)$ implies, for any $N \geq 1$, the following:

$$
\sum_{i=0}^{N-1} r p^{i}=s-s p^{N}
$$

On the left side we have $\operatorname{wd}(r)<d$ and $p$ is homogeneous of degree $d$. Hence there is no cancellation of homogeneous components of the various terms $r p^{i}$ in the sum. Therefore, the equations $(*)$ for all $N \geq 1$ imply first that $r p^{i}=0$ when $\operatorname{ord}(r)+d i>\operatorname{deg}(s)$, and next that $s p^{N}=0$ when $\operatorname{ord}(s)+d N>\operatorname{deg}(s)$.

Proposition 2.9. Assume that a localization $R \subset R\left[S^{-1}\right]$ is countably generated over $R$, where $S \subset R$ is a system of regular elements containing a non-unit. In addition, assume for some element $p \in R$ that the following conditions hold for all non-units $s \in S$ :
(1) $\bigcap_{n} s^{n} R=0$,
(2) $p$ is regular modulo $s$, and
(3) $\bigcap_{i} p^{i}(R / s R)=0$.

Then $R\left[S^{-1}\right]$ is slender.

Proof. Clearly, the conditions (1) and (2) with a regular non-unit $s \in S$ imply that $p$ is a regular non-unit in $R$, and, in fact, a non-unit in $R\left[S^{-1}\right]$. Replacing $S$ by a smaller subset if necessary, we may assume that $S$ is countable and that every $s \neq 1$ in $S$ is a non-unit. Enumerate the elements: $S=\{1\} \cup\left\{s_{0}, s_{1}, s_{2}, \ldots\right\}$, and set $t_{n}:=s_{0} \cdots s_{n-1}\left(s_{0}=1\right)$. Then $t_{n+1}=t_{n} s_{n}$. for $n \geq 0$.

Proceed indirectly and assume that $R^{\prime}:=R\left[S^{-1}\right]$ is nonslender. Then there exists an $R^{\prime}$-linear map $\varphi:\left(R^{\prime}\right)^{\mathbb{N}} \rightarrow R^{\prime}$ with $\varphi_{n} \neq 0$ for all $n$. After a multiplication in the $n^{\prime}$ th coordinate by an element of $S$ and (using (1)) a suitable power of $s_{n}^{-1}$ we may assume that $\varphi_{n} \in R \backslash s_{n} R$.

Let $M_{n} \subseteq R^{\prime}$ be the $R$-submodule $M_{n}:=R / t_{n}$ consisting of fractions $a / t_{n}$ for $a \in R$. Then the $M_{n}$ form an increasing sequence of additive subgroups, and $R^{\prime}=\bigcup_{n} M_{n}$. We prove that there exists a sequence of exponents $i_{0}<i_{1}<\cdots$ such that, with $q_{n}:=p^{i_{n}} / t_{n+1}$, Condition (2.5.1) holds in the following form:

$$
M_{n} \cap\left(q_{n} \varphi_{n}+q_{n+1} R^{\prime}\right)=\emptyset .
$$

As a consequence, by Proposition 2.5, we have $q_{n} \varphi_{n}=0$ for $n \gg 0$. This is the desired contradiction as $q_{n}=p^{i_{n}}$ is regular, and we assumed that $\varphi_{n} \neq 0$ for all $n$.

To determine the sequence $\left(i_{n}\right)$ we note first for any $i$ that $\left(p^{i} / t_{n+1}\right) \varphi_{n} \notin M_{n}$. Indeed, multiplication by $t_{n+1}$ yields the equivalent assertion $p^{i} \varphi_{n} \notin s_{n} R$ which holds since $\varphi_{n} \notin$ $s_{n} R$ and $p$ is regular modulo $s_{n} R$.

Now let $i_{0}:=0$. Recursively, assume that $i_{n}$ is determined. We have just seen that the residue of $p^{i_{n}} \varphi_{n}$ is non-zero in $R / s_{n} R$. Hence, by (3), we may choose $i_{n+1}>i_{n}$ such that the residue of $p^{i_{n}} \varphi_{n}$ is not in $p^{i_{n+1}}\left(R / s_{n} R\right)$, that is,

$$
p^{i_{n}} \varphi_{n} \notin p^{i_{n+1}} R+s_{n} R .
$$

The condition (*) implies the asserted condition $(\dagger)$. Indeed, any element of $R^{\prime}$ may be written in the form $b /\left(t_{n+1} s\right)$ with a suitable element $s \in S$. Hence, a non-empty intersection in $(\dagger)$ will imply an equation of the form:

$$
p^{i_{n}} \varphi_{n} / t_{n+1}+p^{i_{n+1}} b /\left(t_{n+1} s\right)=a / t_{n}
$$


with $a, b \in R$, and multiplication by $t_{n+1}$ yields the equation:

$$
p^{i_{n}} \varphi_{n}+p^{i_{n+1}} b / s=s_{n} a .
$$

The latter equation implies that $p^{i_{n+1}} b \in s R$, and since $p$ is regular modulo $s R$, it follows that $b \in s R$. In other words, the fraction $b / s$ is in $R$. But then ( $\$)$ contradicts $\left(^{*}\right)$. Therefore, the intersection in (†) is empty, and the proof is complete.

Note 2.10. Part of the assumption on $s$ and $p$ in (2) is that $(s, p)$ is a regular sequence (of length 2). In the local Noetherian case, the intersection conditions are automatic, and $(s, p)$ is a regular sequence iff $(p, s)$ is. It is easy to see in general that if the intersection conditions in (1) and (2) are satisfied and $(s, p)$ is a regular sequence, then $(p, s)$ is a regular sequence.

The conditions (1), (2), and (3) are not so rare. Indeed, consider a Noetherian local domain $R$. Let $\mathfrak{p}$ be a prime ideal different from the maximal ideal, and assume that $\mathfrak{p}$ contains a non-zero element $p$ such that $p R$ is a prime ideal. Then the conditions hold for the complement $R \backslash \mathfrak{p}$. But of course, the localization $R_{\mathfrak{p}}$ will normally not be countably generated over $R$.

In fact, the only natural application of 2.9 we have found is to a localization $R \subset R\left[s^{-1}\right]$ generated by a singleton. A typical example is the following result:

Corollary. Let $R_{0}$ be a ring such that $\bigcap_{n} p^{n} R_{0}=0$ for some regular element $p \in R_{0}$. Then the ring of formal Laurent series $R_{0}[[X]]\left[X^{-1}\right]$ is slender.

As a further example, consider the local ring $R:=\mathbb{Z}_{(2)}$, of rational numbers with odd denominators. It is well-known that $R$, as a countable domain, not a field, is slender, cf. 3.10. The localization $R[1 / 2]$, obtained for the singleton $S:=\{2\}$, is the field $\mathbb{Q}$, and hence $\aleph_{0}$-compact. In particular, the localization is nonslender. Note that (1) is satisfied for $R$, but no element $p \in R$ satisfies (2) and (3).

See also Examples 5.8 and 5.10.

Proposition 2.11. If $R$ is complete with respect to a filtration as in Setup 1.1,

$$
\mathfrak{a}_{\bullet}: \quad R \supseteq \mathfrak{a}_{1} \supseteq \mathfrak{a}_{2} \supseteq \cdots, \quad \mathfrak{a}_{n} \neq 0 \text { for all } n, \bigcap \mathfrak{a}_{n}=0,
$$

then $R$ is nonslender.

Assume that there is given a non-discrete Hausdorff filtration with finitely generated ideals,

$$
\mathfrak{b}_{\bullet}: \quad R \supseteq \mathfrak{b}_{1} \supseteq \mathfrak{b}_{2} \supseteq \cdots, \quad \mathfrak{b}_{n} \neq 0 \text { for all } n, \bigcap \mathfrak{b}_{n}=0
$$

Then,

(1) If $R$ is $\aleph_{0}$-compact, then $R$ is complete with respect to the filtration $\mathfrak{b}_{\text {. }}$

(2) If $R$ is nearly compact, then there exists a regular element $u$ such that $R$ is complete

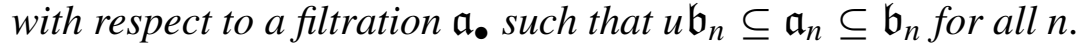

(3) If $R$ is not almost slender, then there exists a sequence of regular elements $p_{n}$ with $p_{n+1} \in p_{n} R$ such that $R$ is complete with respect to the filtration $\left(p_{n} \mathfrak{b}_{n}\right)$.

Proof. The first assertion is well known: $R^{\mathbb{N}}$ is complete with respect to the filtration of "tails" $R^{[n, \infty)}$, and $R$ is complete with respect to $\mathfrak{a}_{\bullet}$. Choose $a_{n} \neq 0$ in $\mathfrak{a}_{n}$ for $n=0,1, \ldots$ Then there is an $R$-linear map of $\mathbb{N}$-systems,

$$
\varphi^{(n)}: R^{\mathbb{N}} / R^{[n, \infty)}=1_{0} R \oplus \cdots \oplus 1_{n-1} R \rightarrow R / \mathfrak{a}_{n},
$$


under which $1_{j} \mapsto a_{j}\left(\bmod \mathfrak{a}_{n}\right)$ for $0 \leq j<n$. Clearly, for the limit, $\varphi=\lim \varphi^{(n)}: R^{\mathbb{N}} \rightarrow$ $R$, we have $\varphi_{n}=a_{n} \neq 0$ for all $n$.

For each of the remaining three assertions part of the hypothesis is the existence of an $R$-linear map $\varphi: R^{\mathbb{N}} \rightarrow R$ with special properties. The submodule $\prod_{n>0} \mathfrak{b}_{n} \subseteq R^{\mathbb{N}}$ is complete with respect to the filtration by the "tails" $\prod_{i=n}^{\infty} \mathfrak{b}_{i}$. Let $\mathfrak{a}_{n}$ denote the image of the tail, $\mathfrak{a}_{n}:=\varphi\left(\prod_{i=n}^{\infty} \mathfrak{b}_{i}\right)$. Then, using an observation of Dimitrić [D, 2.2. Lemma, p. 378], $R$ is is complete with respect to the filtration $\mathfrak{a}_{\bullet}$, provided that the latter filtration is non-discrete Hausdorff.

Clearly, $1_{n} \mathfrak{b}_{n} \subseteq \prod_{i \geq n} \mathfrak{b}_{i} \subseteq \prod_{i \geq n} \mathfrak{b}_{n}=\mathfrak{b}_{n} R^{[n, \infty)}$, where the last equation follows from Lemma 2.12 below. Hence

$$
\varphi_{n} \mathfrak{b}_{n} \subseteq \mathfrak{a}_{n} \subseteq \mathfrak{b}_{n}
$$

In particular, the filtration $\mathfrak{a}_{\bullet}$ is Hausdorff, and non-discrete if $\varphi_{n} \mathfrak{b}_{n} \neq 0$ for all $n$.

Consider first Assertion (2). Here we may assume that $\varphi_{n}=u$ is a constant regular element. So $u \mathfrak{b}_{n} \neq 0$, and (2) has been proved. Moreover, under the hypothesis in (1) we may assume that $\varphi_{n}=1$ for all $n$. that is, $u=1$, and hence $\mathfrak{b}_{\bullet}=\mathfrak{a}_{\bullet}$. Thus (1) is a consequence of (the proof of) (2).

Consider finally (3). There exists an $R$-linear map $\psi: R^{\mathbb{N}} \rightarrow R$ such that $\psi_{n}$ is regular for all $n$. Set $p_{n}:=\psi_{0} \cdots \psi_{n}$, and define $\varphi: R^{\mathbb{N}} \rightarrow R$, for all $x \in R^{\mathbb{N}}$, by

$$
\varphi(x)=\psi\left(x_{0}, p_{0} x_{1}, p_{1} x_{2}, p_{2} x_{3}, \ldots\right)
$$

and, as before, $\mathfrak{a}_{n}:=\varphi\left(\prod_{i \geq n} \mathfrak{b}_{i}\right)$. It suffices to prove that

$$
\mathfrak{a}_{n}=\varphi_{n} \mathfrak{b}_{n}+\varphi\left(\prod_{i>n} \mathfrak{b}_{i}\right)=p_{n} \mathfrak{b}_{n}
$$

since, obviously, the filtration $\left(p_{n} \mathfrak{b}_{n}\right)$ is non-discrete Hausdorff.

The first equality in (2.11.3) follows simply by linearity. As $\varphi_{n}=p_{n-1} \psi_{n}=p_{n}$, to prove the second equality, it suffices to prove for $x \in \prod_{i>n} \mathfrak{b}_{i}$ that $\varphi(x) \in p_{n} \mathfrak{b}_{n}$. For such a sequence $x$, the value $\varphi(x)$ is given by the right side of (2.11.2) where every coordinate belongs to $p_{n} \mathfrak{b}_{n+1}$, and hence to $p_{n} \mathfrak{b}_{n}$. Again, since $\mathfrak{b}_{n}$ is finitely generated, it follows that $\varphi(x) \in p_{n} \mathfrak{b}_{n}$. Hence also (3) has been proved.

Lemma 2.12. Let $\varphi: R^{\mathbb{N}} \rightarrow R$ be a linear map, and $\mathfrak{a} \subseteq R$ a finitely generated ideal. Then $\mathfrak{a} R^{\mathbb{N}}=\mathfrak{a}^{\mathbb{N}}$, and, as a consequence, $\varphi\left(\mathfrak{a}^{\mathbb{N}}\right) \subseteq \mathfrak{a}$ and $\varphi$ induces a linear map $(R / \mathfrak{a})^{\mathbb{N}} \rightarrow R / \mathfrak{a}$.

If $R \supseteq \mathfrak{a}_{1} \supseteq \mathfrak{a}_{2} \supseteq \cdots$ is a descending chain of finitely generated ideals with $\bigcap \mathfrak{a}_{n}=0$, and $\varphi_{n}=0$ for all $n$, then $\varphi$ vanishes on the submodule $\prod \mathfrak{a}_{n} \subseteq R^{\mathbb{N}}$.

Proof. The first assertion is obvious. For the second, note the following inclusions, where $k \geq 1$ is fixed:

$$
\prod_{n \in \mathbb{N}} \mathfrak{a}_{n} \subseteq \prod_{n<k} R \times \prod_{n \geq k} \mathfrak{a}_{n} \subseteq \prod_{n<k} R \times \prod_{n \geq k} \mathfrak{a}_{k}
$$

For the second factor on the right we have $\prod_{n \geq k} \mathfrak{a}_{k}=\mathfrak{a}_{k} \prod_{n \geq k} R$ since $\mathfrak{a}_{k}$ is finitely generated. So, by linearity, $\varphi$ maps the second factor into $\mathfrak{a}_{k}$. Clearly $\varphi$ maps the first factor to zero. Hence $\varphi$ maps the left side $\prod \mathfrak{a}_{n}$ into $\mathfrak{a}_{k}$. Since $k \geq 1$ was arbitrary, it follows that $\varphi$ vanishes on $\prod \mathfrak{a}_{n}$. 


\section{3. "Countability" conditions}

Among domains a field is $\aleph_{0}$-compact. For rings with zero divisors it is a well-known natural generalization that an Artinian ring is $\aleph_{0}$-compact. For a countable domain $R$ it is a classical result, see [E-M, 2.4 Cor., p. 60], that $R$ is slender iff $R$ is a not a field. A substantial generalization of this result is presented in Theorem 3.10. We weaken the countability assumption considerably, see the Definition 3.1 of rings of bounded type. We allow zero divisors, but assume that the nilradical of the ring is finitely generated. Under these assumptions, the ring is almost slender iff it is not Artinian.

In the last subsections we consider for rings of bounded type the question of slenderness. It is much more delicate than the question about almost slenderness.

Definition 3.1. In this section we consider various "countability" conditions on the ring $R$. We say that $R$ is of bounded type if either

(1) $|R|<2^{\aleph_{0}}$, or if

(2) $R$ is an algebra over a field $k$ and $\operatorname{dim}_{k} R<|k|^{\aleph_{0}}$, or, more generally, if

(3) $R$ is an algebra over an Artinian ring $A$ and for every maximal ideal $\mathfrak{m} \subset A$ the dimension of $R / \mathfrak{m} R$ as vector space over $A / \mathfrak{m}$ is strictly less than $|A / \mathfrak{m}|^{\aleph_{0}}$.

Note that "bounded type" includes the following conditions: $R$ is a countable ring $(|R| \leq$ $\left.\aleph_{0}\right)$, or $R$ is countably generated as algebra over a field $k\left(\operatorname{dim}_{k} R \leq \aleph_{0}\right)$, or more generally, $R$ is countably generated over an Artinian ring.

Lemma 3.2. A ring of bounded type is never complete with respect to a non-discrete Hausdorff filtration of ideals $R=\mathfrak{a}_{0} \supset \mathfrak{a}_{1} \supset \cdots$ with $\bigcap \mathfrak{a}_{n}=0$.

Proof. Assume, indirectly, that the canonical map is an isomorphism $R \stackrel{\sim}{\longrightarrow} \lim Q_{n}$, where $Q_{n}=R / \mathfrak{a}_{n}$. Clearly, the transition maps $Q_{n+1} \rightarrow Q_{n}$ in the $\mathbb{N}$-system $\left(Q_{n}\right)$ are surjective, and they are non-injective since the inclusions $\mathfrak{a}_{n+1} \subseteq \mathfrak{a}_{n}$ are strict.

First, for any set $T$ there is an $\mathbb{N}$-system $\left(T^{n}\right)$ with the natural projections $T^{n+1} \rightarrow T^{n}$ as transition maps. Take $T:=\{0,1\}$. Clearly, since every fiber of a transition map $Q_{n+1} \rightarrow Q_{n}$ contains at least two elements, there is an injection of $\mathbb{N}$-systems of sets, $\left(\{0,1\}^{n}\right) \rightarrow\left(Q_{n}\right)$, and hence an injection of the limits, $\{0,1\}^{\mathbb{N}} \rightarrow R$. Thus $2^{\aleph_{0}} \leq|R|$, which is a contradiction under condition 3.1(1).

Similarly, under condition 3.1(2), a $k$-linear injection of $\mathbb{N}$-systems $\left(k^{n}\right) \rightarrow\left(Q_{n}\right)$ is obtained inductively as follows: Lift $k^{n} \rightarrow Q_{n}$ to $k^{n} \rightarrow Q_{n+1}$, and extend to a map from $k^{n+1}=k^{n} \oplus k$ to $Q_{n+1}$ by mapping the last basis vector to a non-zero element in the kernel of $Q_{n+1} \rightarrow Q_{n}$. The result is an injection of the limits, $k^{\mathbb{N}} \rightarrow R$. Thus $\operatorname{dim}_{k} k^{\mathbb{N}} \leq \operatorname{dim}_{k} R$. This a contradiction under condition 3.1(2), since, by the Erdös-Kaplansky theorem (see [J, Chapter IX, §5]), $\operatorname{dim}_{k} k^{\mathbb{N}}=|k|^{\aleph_{0}}$.

The general case where $R$ is an algebra over an Artinian ring $A$ is easily reduced to the case where $A$ is local with, say, maximal ideal $\mathfrak{m}$. We have to prove, with $k:=A / \mathfrak{m}$, that $\operatorname{dim}_{k} R / \mathfrak{m} R \geq \operatorname{dim}_{k} k^{\mathbb{N}}$. It suffices to show that there exists an ideal $J \subseteq R$ such that $\operatorname{dim}_{k} J / \mathfrak{m} J \geq \operatorname{dim}_{k} k^{\mathbb{N}}$. This reduction is possible because $\mathfrak{m} R$ is finitely generated and nilpotent. As we shall show, we may take as $J$ the closure of one of the ideals $\mathfrak{m}^{i} R$.

Fix $i=0,1, \ldots$ The closure of $\mathfrak{m}^{i} R$ is the limit $J_{i}:=\lim _{n} \mathfrak{m}^{i} Q_{n}$ of the subsystem $\left(\mathfrak{m}^{i} Q_{n}\right)$ of $\left(Q_{n}\right)$. Clearly, we have an exact sequence of systems:

$$
0 \rightarrow \mathfrak{m}^{i+1} Q_{n} \rightarrow \mathfrak{m}^{i} Q_{n} \rightarrow \mathfrak{m}^{i} Q_{n} / \mathfrak{m}^{i+1} Q_{n} \rightarrow 0 .
$$

All the transition maps in the systems are surjective. In particular, so are the maps in the 
left system, and thus the resulting left exact sequence of the limits is exact:

$$
0 \rightarrow J_{i+1} \rightarrow J_{i} \rightarrow \underset{n}{\lim }\left(\mathfrak{m}^{i} Q_{n} / \mathfrak{m}^{i+1} Q_{n}\right) \rightarrow 0
$$

Consider the systems (3.2.1) for $i=0$. In the middle system (which is $\left(Q_{n}\right)$ ) the transition maps are non-injective for $n \gg 0$ (in fact, when $n \geq 0$ ). So, if the transistion maps in right system are isomorphisms for $n \gg 0$ then the transition maps in the left system are noninjective for $n \gg 0$. The left system for $i=0$ is the middle system for $i=1$. By repeating this argument, it follows that if the transition maps in the right systems for $i=0, \ldots, p$ are isomorphisms for $n \gg 0$, then the transition maps in the system $\left(\mathfrak{m}^{p+1} Q_{n}\right)$ are noninjective for $n \gg 0$. As this is contradictory when $\mathfrak{m}^{p+1}=0$, we conclude for some $i$ that in the right system in (3.2.1) the transition maps are non-injective for $n \gg 0$.

The right system in (3.2.1) is a system of vector spaces over $k$. As above, it follows from the choice of $i$ that the dimension of the limit is at least $\operatorname{dim}_{k} k^{\mathbb{N}}$. As $J_{i}$ maps surjectively onto this limit we conclude that $\operatorname{dim}_{k} J_{i} / \mathfrak{m} J_{i} \geq \operatorname{dim}_{k} k^{\mathbb{N}}$. Thus the proof is complete.

Corollary 3.3. A ring of bounded type is slender iff it is halfslender.

Proof. The assertion follows immediately from the result of Dimitrić, see Remark 2.3.

Corollary 3.4. Let $R$ be a ring of bounded type, and let $\varphi: R^{\mathbb{N}} \rightarrow R$ be an $R$-linear map. Then the inclusion $\varphi\left(R^{[n+1, \infty)}\right) \subseteq \varphi\left(R^{[n, \infty)}\right)$ is an equality for $n \gg 0$.

Proof. Indeed, set $\mathfrak{a}_{n}:=\varphi\left(R^{[n, \infty)}\right)$ and $\mathfrak{a}:=\bigcap_{n} \mathfrak{a}_{n}$. If the inclusions $\mathfrak{a}_{n+1} \subseteq \mathfrak{a}_{n}$ were strict for infinitely many $n$, then, as noted in Remark 2.3 , the residue ring $R / \mathfrak{a}$ would be complete with respect to the non-discrete Hausdorff filtration $\mathfrak{a}_{n} / \mathfrak{a}$, contradicting Proposition 3.2 since $R / \mathfrak{a}$ is of bounded type.

Corollary 3.5. Let $R$ be a ring of bounded type, and assume that there exists for every non-zero ideal $\mathfrak{a}$ an element $t \in R$ such that $\mathfrak{a} \supset t \mathfrak{a} \supset 0$. Then $R$ is slender.

Proof. Indirectly, assume that $R$ is nonslender. Then, by Corollary 3.4, there exists an $R$-linear map $\varphi: R^{\mathbb{N}} \rightarrow R$ such that all the ideals $\varphi\left(R^{[n, \infty)}\right)$ are non-zero and equal, say $\varphi\left(R^{[n, \infty)}\right)=\mathfrak{a}$ for all $n$.

Now apply the hypothesis repeatedly to obtain a sequence $1=t_{0}, t_{1}, t_{2}, \ldots$ such that

$$
\mathfrak{a} \supset t_{1} \mathfrak{a} \supset t_{2} \mathfrak{a} \supset \cdots, \quad \text { and } t_{n+1} \in t_{n} R \text { for all } n \text {. }
$$

According to the strict inclusions, pick a sequence $a_{0}, a_{1}, a_{2}, \ldots$ of elements of $\mathfrak{a}$ such that $t_{i} a_{i} \notin t_{i+1} \mathfrak{a}$. Then, by Lemma 2.2 , there exists an $R$-linear map $\psi: R^{\mathbb{N}} \rightarrow R$ such that $\psi_{n}=a_{n}$ and $\psi\left(R^{[n, \infty)}\right) \subseteq \mathfrak{a}$ for all $n$. Finally, define $\chi: R^{\mathbb{N}} \rightarrow R$ as the following modification of $\psi$ :

$$
\chi(x)=\psi\left(x_{0}, t_{1} x_{1}, t_{2} x_{2}, \ldots\right) .
$$

Clearly, if $x \in R^{[n, \infty)}$ then all the arguments on the right side of (3.5.1) belong to $t_{n+1} R$. Hence,

$$
\chi\left(R^{[n+1, \infty)}\right) \subseteq t_{n+1} \psi\left(R^{[n+1, \infty)}\right) \subseteq t_{n+1} \mathfrak{a} .
$$

So the choice of $a_{n}$ implies that $t_{n} a_{n} \notin \chi\left(R^{[n+1, \infty)}\right)$. However, $t_{n} a_{n}=\chi_{n} \in \chi\left(R^{[n, \infty)}\right)$. Therefore, the inclusion $\chi\left(R^{[n+1, \infty)}\right) \subseteq \chi\left(R^{[n, \infty)}\right)$ is strict. As this is in conflict with Corollary 3.4, the desired contradiction has been established. 
Proposition 3.6. Let $R$ be a ring of bounded type. Assume in addition any of the following three conditions:

(1) $R$ is reduced and without simple ideals,

(2) $R$ is Noetherian and without simple ideals,

(3) There exists a decreasing sequence $R=q_{0} R \supseteq q_{1} R \supseteq q_{2} R \supseteq \cdots$ with regular elements $q_{j}$ and with $\bigcap_{j} q_{j} R=0$.

Then $R$ is slender.

Proof. We prove under any of the conditions that the assumption in Corollary 3.5 is satisfied. So let $\mathfrak{a}$ be a non-zero ideal.

Case (1): Pick a non-zero element $a \in \mathfrak{a}$, The ideal $a R$ is not simple. In particular, we may assume that $a R \subset \mathfrak{a}$. Then $a \mathfrak{a} \subseteq a R \subset \mathfrak{a}$, and $a \mathfrak{a} \neq 0$ since $a^{2} \in a \mathfrak{a}$ and $R$ is reduced.

Case (2): As $R$ is Noetherian, we may first pick $a R$ maximal among the principal ideals contained in $\mathfrak{a}$, and next, since $a R$ is not simple, choose $t$ such $a R \supset \operatorname{taR} \supset 0$. Then $t \mathfrak{a} \neq 0$, since $t a \neq 0$, and $\mathfrak{a} \supset t \mathfrak{a}$, since the maximality of $a R$ implies that $a \notin t \mathfrak{a}$.

Case (3): Pick any non-zero $a \in \mathfrak{a}$, and choose $n$ such that $a \notin q_{n} R$. Then $q_{n} \mathfrak{a} \neq 0$ since $q_{n}$ is regular, and the inclusion $q_{n} \mathfrak{a} \subseteq \mathfrak{a}$ is strict, since $a \notin q_{n} \mathfrak{a}$.

Thus $R$ is slender in all three cases.

Remark 3.7. The conclusion under the first condition is the commutative version of a result of El Bashir and Kepka [EB-K1, Theorem 5.1, p. 1578]. However, their proof requires certain hypotheses on the minimal primes of $R$. The conclusion under the second condition is an immediate consequence of a strong result of El Bashir and Barka [BK-2, 4.4 Corollary, p. 2590] that a Noetherian ring $R$ without simple ideals is nonslender iff $R$ is complete with respect to some non-discrete Hausdorff filtration.

We prove in Lemma 6.7 for an arbitrary Noetherian ring that Condition (3) is a conseqence of (2), and we use it to derive the result of El Bashir and Barka, see Theorem 6.8.

Definition 3.8. The commutative ring $R$ will be called perfect if there does not exist an infinite strictly decreasing chain of principal ideals,

$$
R \supset c_{1} R \supset c_{2} R \supset \cdots .
$$

Note that this condition is one of several equivalent conditions formulated for noncommutative rings by Bass [Ba, Theorem P, p. 467].

Lemma 3.9. If $R$ is of bounded type and not perfect, then $R$ is almost slender.

Proof. Assume, indirectly, that there exists an $R$-linear map $\varphi: R^{\mathbb{N}} \rightarrow R$ such that $\varphi_{n}$ is regular for all $n$. By hypothesis there exists an infinite chain (3.8.1). We will construct a modification of $\varphi$ that violates 3.4.

Set $p_{n}:=\varphi_{1} \cdots \varphi_{n}$ for $n=1,2, \ldots$ As each $\varphi_{i}$ is regular, so is their product $p_{n}$. Consider the modification,

$$
\psi(x)=\varphi\left(x_{0}, c_{1} x_{1}, p_{1} c_{2} x_{2}, p_{2} c_{3} x_{3}, \ldots\right) .
$$

If $x \in R^{[n+1, \infty)}$, then all coordinates on the right side are divisible by $p_{n} c_{n+1}$, and hence

$$
\psi\left(R^{[n+1, \infty)}\right) \subseteq p_{n} c_{n+1} R .
$$

On the other hand, we have $\psi_{n}=p_{n-1} c_{n} \varphi_{n}=p_{n} c_{n}$. Choose $y \in R$ such that $y \in c_{n} R$ and $y \notin c_{n+1} R$. As $p_{n}$ is regular, it follows that $p_{n} y \in p_{n} c_{n} R$ and $p_{n} y \notin p_{n} c_{n+1} R$. Thus $p_{n} y \in R \psi_{n}$ belongs to $\psi\left(R^{[n, \infty)}\right)$, but $p_{n} y \notin \psi\left(R^{[n+1, \infty)}\right)$. 
Theorem 3.10. Assume that $R$ is of bounded type. Then the following three conditions are equivalent:

(i) $R$ is $\aleph_{0}$-compact,

(ii) $R$ is nearly compact,

(iii) $R$ is not almost slender,

and they imply the following condition:

(iv) $R$ is perfect.

If the nilradical $\mathfrak{n}$ of $R$ is finitely generated, then all four conditions are equivalent, and they hold iff $R$ is Artinian.

Proof. The implications (i) $\Rightarrow$ (ii) $\Rightarrow$ (iii) (for any ring) are obvious from the definitions. The implication (iii) $\Rightarrow$ (iv) follows directly from Lemma 3.9. Moreover, if (iv) holds, then every regular element is invertible, and hence (iii) $\Rightarrow$ (i). Hence the first part of the Corollary has been proved.

Assume in addition that $\mathfrak{n}$ is finitely generated. As is well known (see Theorem 6.3), if $R$ is Artinian then (i), and hence all the conditions hold. Conversely, we assume that (iv) holds, and prove that $R$ is Artinian. Again, the assertion results from Bass [Ba, Theorem $\mathrm{P}$, " $(6) \Rightarrow(1) "$, p. 467]. Indeed, it follows first that $R / \mathfrak{n}$ is a finite product of fields, and since the nilradical $\mathfrak{n}$ is nilpotent, say $\mathfrak{n}^{N}=0$, the ring $R$ has a finite filtration whose residue modules $\mathfrak{n}^{i-1} / \mathfrak{n}^{i}$ for $i=1, \ldots, N$ are finitely generated over $R / \mathfrak{n}$. Thus each residue module has finite length. Hence, so has $R$.

Proposition 3.11. If $R$ has a field as a direct factor, then $R$ is nonslender. Conversely, assume that $R$ is of bounded type, reduced, and nonslender. Then $R$ has a field as a direct factor.

Proof. The final conclusion is the commutative version of a result of El Bashir and Kepka [EB-K1, Theorem 5.1, p. 1578]. However, their proof requires certain hypotheses on the minimal primes of $R$.

The first assertion is obvious; in fact, the conclusion holds if $R$ has a direct factor which is nonslender.

Conversely, assume that $R$ is of bounded type, reduced, and nonslender. Then, by Proposition 3.6, $R$ has a simple ideal, That ideal, in a reduced ring, is generated by an idempotent $e \in R$, corresponding to a factor $R / \operatorname{Ann}(e)$ which is a field.

Theorem 3.12. Assume that $R$ is of bounded type, and that the nilradical $\mathfrak{n}$ of $R$ is finitely generated. Then the following two conditions are equivalent:

(i) $R$ has a non-zero Artinian ring as direct factor.

(ii) There exists an $R$-linear map $\varphi: R^{\mathbb{N}} \rightarrow R$ such that no $\varphi_{n}$ is nilpotent.

Proof. Assume (i). As is well known, an Artinian ring is $\aleph_{0}$-compact, see Theorem 6.3. Hence, if $R=A \times S$ with an Artinian factor $A$, then there exist an $R$-linear map $\varphi$ with $\varphi_{n}=(1,0) \in A \times S$ for all $n$. In particular, no $\varphi_{n}$ is nilpotent.

Conversely, assume (ii). Let $\bar{R}:=R / \mathfrak{n}$ be the residue ring. The map $\varphi$ induces, since $\mathfrak{n}$ is finitely generated, an $\bar{R}$-linear map $\bar{\varphi}: \bar{R}^{\mathbb{N}} \rightarrow \bar{R}$, and $\bar{\varphi}_{n} \neq 0$ for all $n$ since $\varphi_{n} \notin \mathfrak{n}$. Hence $\bar{R}$ is nonslender, and, obviously, reduced.

Therefore, by Proposition 3.6(1), $\bar{R}$ has a simple ideal, and as $\bar{R}$ is reduced it follows that the simple ideal splits off as a field, that is, we have a decomposition $\bar{R}=K \times S$ with a field $K$. The hypothesis implies that $\mathfrak{n}$ is nilpotent, say $\mathfrak{n}^{\mathbb{N}}=0$. Consequently the decomposition lifts to a decomposition $R=A \times T$ such that $K=A / \mathfrak{n}(A)$ and $S=T / \mathfrak{n}(T)$. The 
nilradicals $\mathfrak{n}(A)$ and $\mathfrak{n}(T)$ are the images of $\mathfrak{n}(R)$ under the two projections. In particular, $\mathfrak{n}(A)$ is finitely generated and $\mathfrak{n}(A)^{N}=0$. So we obtain a finite filtration of $A$ whose residue modules $\mathfrak{n}(A)^{i-1} / \mathfrak{n}(A)^{i}$ for $i=1, \ldots, N$ are finite dimensional over $K$. Hence $A$ has finite length.

\section{Von Neumann regular rings and valuation rings}

In this section we use our previous results to study slenderness and compactness for von Neumann regular rings and valuation rings. Note that, by Proposition 3.6(1), a von Neumann regular ring $R$ of bounded type is slender iff $R$ contains no simple ideals.

Definition 4.1. In this section we will say that a sequence $a=\left(a_{n}\right)$ in $R^{\mathbb{N}}$ is divisorial if, among the principal ideals containing $a_{n}$ for all $n$, there is a smallest. In other words, $a=\left(a_{n}\right)$ is divisorial if there exists an element $d \in R$ such that for all elements $c \in R$ we have $a_{n} \in c R$ for all $n$ iff $d R \subseteq c R$. We keep the notion of a nonslender sequence introduced in Definition 3.11.

Lemma 4.2. Any nonslender sequence $e \in R^{\mathbb{N}}$ of pairwise orthogonal idempotents is divisorial.

Proof. As the sequence $e$ is nonslender there is a linear map $\varphi: R^{\mathbb{N}} \rightarrow R$ such that $\varphi_{n}=e_{n}$ for all $n$. Set

$$
d:=\varphi(e) \text {. }
$$

The entries in $e$ are orthogonal idempotents. Hence, for every $n \geq 0$, we have that $e_{n} d=$ $e_{n} \varphi(e)=\varphi\left(e_{n}^{2} 1_{n}\right)=e_{n}^{3}=e_{n}$; in particular, $e_{n} \in d R$. Moreover, if $e_{n} \in c R$ for all $n$ then $e \in c R^{\mathbb{N}}$; hence, by linearity, $d=\varphi(e) \in c R$.

Lemma 4.3. If $R$ is $\aleph_{0}$-compact, then every sequence of pairwise orthogonal non-zero idempotents is divisorial.

Proof. Indeed, if $R$ is $\aleph_{0}$-compact, then any sequence in $R^{\mathbb{N}}$ with non-zero entries is nonslender.

Lemma 4.4. If $R$ is von Neumann regular and some sequence of non-zero orthogonal idempotents is not divisorial, then $R$ is almost slender.

Proof. Assume, indirectly, that $R$ is not almost slender. In a von Neumann regular ring every regular element is invertible. Hence, $R$ is $\aleph_{0}$-compact. By hypothesis there exists a sequence of non-zero orthogonal idempotents which is not divisorial, in contrast to the conclusion in Lemma 4.3.

Proposition 4.5. Consider for a von Neumann regular ring $k$ the product ring $k^{\mathbb{N}}$, and view $k^{(\mathbb{N})}$ as an ideal in $k^{\mathbb{N}}$. Then the residue ring $R:=k^{\mathbb{N}} / k^{(\mathbb{N})}$ is slender.

Proof. The assertion for a general von Neumann regular ring $k$ rather than a field was kindly communicated to us by the referee. The proof below is inspired by the remarks of the referee, see also [EB-K3, Proposition 7.1, p. 284].

Assume, indirectly, that $R$ is nonslender. Then there exists an $R$-linear map $\varphi: R^{\mathbb{N}} \rightarrow R$ such that $\varphi_{n} \neq 0$ for all $n$. If $a \mapsto \bar{a}$ denotes the residue map $k^{\mathbb{N}} \rightarrow R$, we have $\varphi_{n}=\bar{e}_{n}$ for elements $e_{n} \in k^{\mathbb{N}}$. As $R$ is von Neumann regular, we may assume that each $\bar{e}_{n}$ is idempotent, and obviously, we may assume that each lifted sequence $e_{n} \in k^{\mathbb{N}}$ is idempotent too. As $\bar{e}_{n} \neq 0$, we have $e_{n} \not \equiv 0$, where the congruence is modulo $k^{(\mathbb{N})}$.

We will first prove that there exists a sequence of idempotents $f_{n} \in k^{\mathbb{N}}$ whose supports are pairwise disjoint and such that the sequence $\left(\bar{f}_{n}\right)$ in $R^{\mathbb{N}}$ is nonslender. Indeed, decompose 
$\mathbb{N}$ in two disjoint subsets, $\mathbb{N}=A \cup B$, such that each of $A$ and $B$ has an infinite intersection with the support of $e_{0}$. Thus $e_{0} 1_{A} \not \equiv 0$ and $e_{0} 1_{B} \not \equiv 0$. As $1_{A}+1_{B}=1_{\mathbb{N}}$ we have either $e_{j} 1_{A} \not \equiv 0$ for infinitely many $j \geq 1$ or $e_{j} 1_{B} \not \equiv 0$ for infinitely many $j \geq 1$. If the last case does not occur, we interchange $A$ and $B$. Then, after a restriction to an infinite subset of indices, we may assume that $e_{j} 1_{B} \not \equiv 0$ for all $j \geq 1$. Now set $f_{0}:=e_{0} 1_{A}$ and replace $e_{j}$ by $e_{j} 1_{B}$ for $j \geq 1$. Apply the same process to $e_{1}$ and the $e_{j}$ for $j>1$ to obtain $f_{1}$, and continue. Clearly, we obtain a sequence $\left(f_{n}\right)$ as required.

The sequence $\left(\bar{f}_{n}\right)$ in $R^{\mathbb{N}}$ is a nonslender sequence of pairwise orthogonal idempotents. We finish the proof by showing that this sequence is not divisorial, in contrast to the conclusion in Lemma (4.2).

Assume for some $d \in k^{\mathbb{N}}$ that $\bar{f}_{i} \in \bar{d} R$ for all $i$. Let $T$ be the support of $d$, and let $S_{i}$ be the support of $f_{i}$. The relation $\bar{f}_{i} \in \bar{d} R$ implies that the set $S_{i}$, except for finitely many elements, is contained in $T$. In particular, we may choose an index $u_{i} \in S_{i} \cap T$. The $S_{i}$ are pairwise disjoint, so the set $U:=\left\{u_{0}, u_{1}, \ldots\right\}$ is infinite. Multiply $d$ and the $f_{i}$ by the characteristic function $1_{\mathbb{N}-U}$. In the product $h:=d 1_{\mathbb{N}-U}$ the infinitely many coordinates in $U$ are changed to zeros, so that $\bar{h} R \subset \bar{d} R$, but the multiplication changes in $f_{i}$ only a single coordinate. Hence the residue $\bar{f}_{i}$ is unchanged, and it follows that $\bar{f}_{i} \in \bar{h} R$ for all $i$. Thus there is no smallest principal ideal containing the $\bar{f}_{i}$.

Corollary 4.6. Let $R$ be a ring containing a finitely generated maximal ideal. Then the residue ring $R^{\mathbb{N}} / R^{(\mathbb{N})}$ is not $\aleph_{0}$-compact.

Proof. Let $\mathfrak{m} \subset R$ be a finitely generated maximal ideal. We will apply Proposition 4.5 to the residue field $k:=R / \mathfrak{m}$. To simplify the notation, let $P:=R^{\mathbb{N}}$ be the product ring, and $I:=R^{(\mathbb{N})}$ the ideal in $P$. Then the ring in question is the residue ring $Q:=P / I$. The ideal $\mathfrak{m} Q \subseteq Q$ is finitely generated. Hence, to prove that $Q$ is not $\aleph_{0}$-compact, it suffices to prove that $Q / \mathfrak{m} Q$ is not $\aleph_{0}$-compact.

Now there is an exact sequence,

$$
0 \rightarrow I / \mathfrak{m} I \rightarrow P / \mathfrak{m} P \rightarrow Q / \mathfrak{m} Q \rightarrow 0 .
$$

Indeed, the sequence is right exact, and exactness follows from the equality $I \cap \mathfrak{m} P=\mathfrak{m} I$. Again, since $\mathfrak{m} \subset R$ is finitely generated we have the equations: $I / \mathfrak{m} I=(R / \mathfrak{m})^{(\mathbb{N})}$ and $P / \mathfrak{m} P=(R / \mathfrak{m})^{\mathbb{N}}$. Hence the exact sequence identifies $Q / \mathfrak{m} Q$ with the residue ring $k^{\mathbb{N}} / k^{(\mathbb{N})}$. So, by Proposition 4.5, $Q / \mathfrak{m} Q$ is slender, and, in particular, not $\aleph_{0}$-compact.

Setup 4.7. Let $V$ be a DVR (discrete (rank 1) valuation ring), with maximal ideal $\mathfrak{m}_{V}$ and parameter $p$, that is, $\mathfrak{m}_{V}=p V$. Let $T \subset V$ be a system of representatives for the residue field $V / \mathfrak{m}_{V}$. Clearly for every element $x \in V$ there is, for any $n \geq 1$ a congruence,

$$
x \equiv t_{0}+t_{1} p+\cdots+t_{n} p^{n} \quad\left(\bmod p^{n+1} V\right),
$$

with coefficients $t_{j} \in T$ for $j=0, \ldots, n$. The coefficient $t_{j}$ is uniquely determined, and independent of $n$ for $n \geq j$.

Let $V \subseteq V^{\prime}$ be an inclusion of DVRs such that $V^{\prime}$ is finitely generated as module over $V$. The inclusion $V \subseteq V^{\prime}$ is local since, by Nakayama's Lemma, $\mathfrak{m}_{V} V^{\prime} \subseteq \mathfrak{m}_{V^{\prime}}$. Hence, if $p$ and $p^{\prime}$, respectively, are the parameters for $V$ and $V^{\prime}$, we have $p V^{\prime}=\left(p^{\prime}\right)^{e} V^{\prime}$ where $e=e_{V^{\prime} / V} \geq 1$ is the ramification index of $V^{\prime} / V$. Let $f=f_{V^{\prime} / V}$ denote the residue degree of the inclusion, that is, the field degree of $V^{\prime} / p^{\prime} V^{\prime}$ over $V / p V$. Again, by Nakayama's Lemma, we have ef $=1$, iff $V=V^{\prime}$. It should be mentioned that the setup here: $V^{\prime}$ is a DVR, and finitely generated as $V$-module, is natural when $V$ is a complete DVR, and possibly in no other cases. Anyway, in this setup it follows easily that $V^{\prime}$ is a free $V$-module, and that its rank is equal to the product $e_{V^{\prime} / V} f_{V^{\prime} / V}$. 
Proposition 4.8. Assume that $R$ is the union of a strictly increasing sequence of DVR's $V_{0} \subset V_{1} \subset \cdots$ such that each $V_{n+1}$ is finitely generated over $V_{n}$. Then $R$ is slender.

Proof. Assume, indirectly, that there exists an $R$-linear map $\varphi: R^{\mathbb{N}} \rightarrow R$ such that $\varphi_{n} \neq 0$ for all $n$. Rather than replacing the sequence $\left(V_{n}\right)$ by a subsequence, we may assume that $\varphi_{n} \in V_{n}$ for all $n$. Then $\varphi_{n}$ is a unit times a power of the parameter $p_{n}$ for $V_{n}$. After a multiplication in each coordinate of $\varphi$ by a unit, we may assume that $\varphi_{n}=p_{n}^{i_{n}}$ with $i_{n} \geq 0$. We will prove that there is, after a restriction to a subsequence of indices, a sequence of non-zero elements $q_{n}$ such that $q_{n+1} \in q_{n} R$, and such that the following equations hold:

$$
\left(q_{n} \varphi_{n}+q_{n+1} R\right) \cap V_{n}=\emptyset \text { for all } n .
$$

From these equations it follows, by Proposition 2.5, that $q_{n} \varphi_{n}=0$ for $n \gg 0$, contradicting that $\varphi_{n}$ and $q_{n}$ were assumed to be non-zero for all $n$.

The proof of Equation (4.8.1) is divided in two cases depending on the sequence $e_{n}:=$ $e_{V_{n+1} / V_{n}}$.

Case 1: $e_{n}=1$ for $n \gg 0$ : Omitting a finite number of indices, we may assume that $e_{n}=1$ for all $n$. Then the parameter $p=p_{0}$ for $V_{0}$ is a parameter for every $V_{n}$, and we may assume that $\varphi_{n}=p^{i_{n}}$; in particular, $\varphi_{n} \in V_{0}$.

Clearly, we may choose for every $n$ a system $U_{n} \subset V_{n}$ of representatives for the set of non-zero elements of the residue field $V_{n} / p V_{n}$ in such a way that $U_{n} \subset U_{n+1}$; the inclusion is strict since $f\left(V_{n+1} / V_{n}\right)>1$. Assume that 1 belongs to $U_{0}$. Define the sequence $q_{n}$ of the form $q_{n}=u_{n} p^{k_{n}}$ recursively on the pairs $u_{n}, k_{n}$ with $u_{0}=1, k_{0}=0$, and

$$
u_{n} \in U_{n+1} \backslash U_{n}, \quad k_{n+1}>i_{n}+k_{n} .
$$

Note that $q_{n+1} \in q_{n} R$ since $k_{n+1}>k_{n}$ and $u_{n+1}$ is invertible. To verify (4.8.1) let $x \in q_{n} \varphi_{n}+q_{n+1} R$, say $x=u_{n} p^{i_{n}+k_{n}}+p^{k_{n+1}} y$. Then it follows from the uniqueness in (4.7.1) and the relations in (4.8.2) that $x \notin V_{n}$. Thus (4.8.1) holds, and the proof in Case 1 is complete.

Case 2: $e_{n}>1$ for infinitely many $n$ : Restricting to a subsequence of indices we may assume that $e_{n}>1$ for all $n$. We have $\varphi_{n}=p_{n}^{i_{n}}$. Define the sequence $q_{n}$ of the form $q_{n}=p_{n+1}^{k_{n}}$ recursively on the exponents $k_{n}$ :

$$
e_{n} \backslash k_{n} \text { and } k_{n+1} \geq\left(k_{n}+i_{n} e_{n}+1\right) e_{n+1} .
$$

Note that $q_{n+1} \in q_{n} R$ since $k_{n+1} \geq k_{n} e_{n+1}$. To verify (4.8.1), fix $n$, and let $x$ be an element of the form $x=q_{n} \varphi_{n}+q_{n+1} y, y \in R$. Set $h:=k_{n}+i_{n} e_{n}$. As $p_{n}$ is a unit times $p_{n+1}^{e_{n}}$ we get $q_{n} \varphi_{n}=u p_{n+1}^{h}$ with a unit $u \in V_{n+1}$. In addition, since $k_{n+1} \geq(h+1) e_{n+1}$ we have $q_{n+1}=p_{n+2}^{k_{n+1}} \in p_{n+1}^{h+1} R$. Hence we have

$$
x=u p_{n+1}^{h}+p_{n+1}^{h+1} s, \quad s \in R .
$$

It follows from (4.8.3) that $e_{n} \backslash h$. Therefore, by the uniqueness in (4.7.1), $x \notin V_{n}$. Thus (4.8.1) holds, and the proof in Case 2 is complete.

Lemma 4.9. If a valuation ring with countable value group is $\aleph_{0}$-compact, then it is algebraically compact.

Proof. Indeed, such a ring is, in particular, coherent and has at most $\aleph_{0}$ finitely generated ideals. Hence the assertion follows from [J-L, Ex. 7.30, p. 145]. 
Example 4.10. As a first example, let $R$ be the ring of (integral) Puiseux series over a field $k$, defined as the directed union of $k\left[\left[X^{1 / n}\right]\right]$ for integers $n \geq 1$. The elements of $R$, and of the rings described below, may be viewed as formal power series $\sum_{\alpha} a_{\alpha} X^{\alpha}$, indexed by non-negative rational numbers $\alpha$. Such a series belongs to $R$, if its support, that is, the set of indices $\alpha$ such that $a_{\alpha} \neq 0$, is contained in $(1 / n) \mathbb{Z}$ for some $n \geq 1$. By Puiseux's theorem, if $k$ is algebraically closed of characteristic zero, then the fraction field of $R$ is algebraically closed.

The ring $R$ is slender by Proposition 4.8, and thus, in particular, cf. Proposition 2.11, $R$ is not $X$-adically complete.

The $X$-adic completion $\widehat{R}$ consists of the formal power series $\sum_{\alpha} a_{\alpha} X^{\alpha}$ for which the support contains only finitely many indices from any bounded interval. The ring $\widehat{R}$ is a valuation ring; its value group is $\mathbb{Q}$, and its residue field is $k$. As a completion, $\widehat{R}$ is nonslender by Proposition 2.11. We will show that $\widehat{R}$ is not $\aleph_{0}$-compact.

Let $S \supset R$ be the ring consisting of the formal series $\sum_{\alpha} a_{\alpha} X^{\alpha}$ for which the support is a well-ordered subset of $\mathbb{Q}$. Again, $S$ is a valuation ring with value group $\mathbb{Q}$ and residue field $k$. Hence $S$ is a proper immediate extension of $\widehat{R}$, and, as a consequence, $\widehat{R}$ is not maximally complete. Therefore, by [J-L, Theorem 11.18, p. 289] $\widehat{R}$ is not algebraically compact. Since the value group $\mathbb{Q}$ is countable, it follows from Lemma 4.9 that $\widehat{R}$ is not $\aleph_{0}$-compact.

Example 4.11. The integral closure, $\overline{\mathbb{Z}}_{p}$, of the ring $\mathbb{Z}_{p}$ of $p$-adic integers is slender. This result follows from 4.8 by using Krasner's result that the integral closure $\overline{\mathbb{Z}}_{p}$ is a countable union of finite extensions $V / \mathbb{Z}_{p}$. Note that, by Proposition 2.11 , the slenderness of $\overline{\mathbb{Z}}_{p}$ implies that $\overline{\mathbb{Z}}_{p}$ is not complete in the $p$-adic topology; the argument given here is an alternative to the proof in [G, Theorem 5.7.5, p. 165].

For the following we refer to [G, p. 168ff.]. The $p$-adic completion of $\overline{\mathbb{Z}}_{p}$, denoted $V_{p}$, is a valuation ring with value group $\mathbb{Q}$; its fraction field, often denoted $C_{p}$, is algebraically closed. As a completion, $V_{p}$ is nonslender. An argument as in the previous example shows that $V_{p}$ is not $\aleph_{0}$-compact. Indeed, it may be shown that $V_{p}$ admits a proper immediate extension, cf. B. Poonen [P, p. 98], and it follows first that $V_{p}$ is not algebraically compact, and next by Lemma 4.9 that $V_{p}$ is not $\aleph_{0}$-compact.

Setup 4.12. Fix an infinite set $J$, and let $R_{\alpha}, \alpha \in J$, be a $J$-indexed family of algebras over a ring $k$ such that all the structure maps $k \rightarrow R_{\alpha}$ are injective. An obvious example is to take algebras over a field $k$.

Consider the product ring $\prod_{\alpha} R_{\alpha}$. A family $r=\left(r_{\alpha}\right)$ is called eventually constant if there exists an element $\lambda \in k$ such that $r_{\alpha}=\lambda$ except for the indices in a subset of cardinality strictly smaller than $|J|$. The constant $\lambda$ is then unique, denoted $r_{\infty}$. The subring $R$ of eventually constant families is denoted as follows:

$$
R:=\prod_{\alpha}^{\prime} R_{\alpha} \subset \prod_{\alpha} R_{\alpha}
$$

For any index $\alpha \in J$ consider the $k$-linear embedding $\delta_{\alpha}: R_{\alpha} \hookrightarrow \prod_{\beta} R_{\beta}$. The image of $w \in R_{\alpha}$ is the family $w \delta_{\alpha}$ which is $w$ in coordinate $\alpha$ and 0 at all other coordinates. In particular, $w \delta_{\alpha}$ is eventually 0 and hence $w \delta_{\alpha} \in R$. So, the embeddings $\delta_{\alpha}: R_{\alpha} \rightarrow R$ for $\alpha \in J$ define a (diagonal) embedding,

$$
\delta: \prod_{\alpha} R_{\alpha} \hookrightarrow R^{J}
$$


Lemma 4.13. In the notation of 4.12 the ring $R:=\prod_{\alpha}^{\prime} R_{\alpha}$ is almost $J$-slender.

Proof. In other words, there is no $R$-linear map $\varphi: R^{J} \rightarrow R$ such that $\varphi\left(1_{\alpha}\right)$ is regular in $R$ for all $\alpha \in J$.

Assume indirectly that $\varphi$ is such a map. Let $J=J^{\prime} \cup J^{\prime \prime}$ be a decomposition such at $J^{\prime} \cap J^{\prime \prime}=\emptyset$ and $\left|J^{\prime}\right|=\left|J^{\prime}\right|=|J|$.

Let $\chi \in \prod_{\alpha} R_{\alpha}$ be the family such that $\chi_{\alpha}=1$ for $\alpha \in J^{\prime}$ and $\chi_{\alpha}=0$ for $\alpha \in J^{\prime \prime}$. Form the family $\chi \delta \in R^{J}$ and consider the values,

$$
r:=\varphi(\chi \delta) \in R \text { and } \lambda:=r_{\infty}
$$

Let $\beta \in J$ be an index such that $r_{\beta}=\lambda$. By the choice of $J^{\prime}$ and $J^{\prime \prime}$ there is at least one such $\beta$ in $J^{\prime}$ and one in $J^{\prime \prime}$. Let $w \in R_{\beta}$ be arbitrary. Then $\left(w \delta_{\beta}\right) \in R$, and

$$
\left(w \delta_{\beta}\right) \varphi(\chi \delta)=\left(w \delta_{\beta}\right) r=(w \lambda) \delta_{\beta} .
$$

By linearity,

$$
\left(w \delta_{\beta}\right) \varphi(\chi \delta)=\varphi\left(w \chi_{\beta} \delta_{\beta}\right)=\left(w \chi_{\beta} \delta_{\beta}\right) \varphi_{\beta} .
$$

The value $\varphi_{\beta}=\varphi\left(1_{\beta}\right)$ is regular in $R$. In particular, the $\beta$ 'th coordinate $\varphi\left(1_{\beta}\right)_{\beta}$ is non-zero in $R_{\beta}$.

Equate the two right sides in (1) and (2), and take the coordinate corresponding to $\beta$, to obtain the equations in $R_{\beta}$,

$$
w \lambda=w \chi_{\beta} \varphi\left(1_{\beta}\right)_{\beta}, \quad \lambda=\chi_{\beta} \varphi\left(1_{\beta}\right)_{\beta} .
$$

The second equation is obtained from the first by setting $w=1 \in R_{\beta}$.

Now, if $\beta \in J^{\prime \prime}$ we have $\chi_{\beta}=0$, and we conclude that $\lambda=0$. If $\beta \in J^{\prime}$ we have $\chi_{\beta}=1$, and we conclude that $\lambda \neq 0$. This is the desired contradiction.

Lemma 4.14. In the notation of 4.12, assume that $|J|>\aleph_{0}$. Then, if each $R_{\alpha}$ is $\aleph_{0^{-}}$ compact, then so is $R$.

Proof. By assumption there exists for each $\alpha \in J$ an $R_{\alpha}$-linear map $\varphi_{\alpha}: R_{\alpha}^{\mathbb{N}} \rightarrow R_{\alpha}$ such that $\varphi_{\alpha}\left(1_{n}\right)=1$ for all $n$. Consider the product map,

$$
\prod_{\alpha} \varphi_{\alpha}:\left(\prod_{\alpha} R_{\alpha}\right)^{\mathbb{N}}=\prod_{\alpha} R_{\alpha}^{\mathbb{N}} \rightarrow \prod_{\alpha} R_{\alpha}
$$

and check that it induces the required $R$-linear map,

$$
\left(\prod_{\alpha}^{\prime} R_{\alpha}\right)^{\mathbb{N}} \rightarrow \prod_{\alpha}^{\prime} R_{\alpha}
$$

Example 4.15. With $R_{\alpha}:=k$ ( $k$ a field) and $|J|=\aleph_{1}$ we obtain a von Neumann regular ring,

$$
R:=\prod_{\alpha \in J}^{\prime} k \subseteq k^{J} .
$$

The ring $R$ is $\aleph_{0}$-compact by Lemma 4.14; in particular, $R$ is not almost slender. By Lemma 4.13, $R$ is almost $\aleph_{1}$-slender; in particular, $R$ is not $\aleph_{1}$-compact.

We know of no example of a domain which is $\aleph_{0}$-compact but not $\aleph_{1}$-compact. 


\section{Examples and minor results}

Example 5.1. Consider the power series ring $R:=\mathbb{Z}_{(p)}[[X]]$ over the local ring $\mathbb{Z}_{(p)}$ of rationals with denominators not divisible by the prime number $p$. The ring $R$ is local Noetherian domain of dimension 2. Moreover $R$ is neither slender, nor nearly compact. This is a consequence of Propositions 2.4(b) and 2.7. For the details, see [JJT, Example 3.2, p. 900].

Example 5.2. Let $V$ be a vector space over the field $k$, and let $R=k \oplus V$ be the $k$-algebra with multiplication given by $V \cdot V=0$. We prove that $R$ is $\aleph_{0}$-compact. Clearly,

$$
R^{\mathbb{N}}=k^{\mathbb{N}} \oplus V^{\mathbb{N}}
$$

Since a field is $\aleph_{0}$-compact, there exists a $k$-linear map $\varphi: k^{\mathbb{N}} \rightarrow k$ such that $\varphi_{n}=1$ for all $n$. The map $\varphi$ is extended to an $R$ linear map $\varphi: R^{\mathbb{N}} \rightarrow R$ as follows: Assume first that $V$ is of finite dimension, say $V=k e_{1} \oplus \cdots \oplus k e_{d}$. Then clearly,

$$
V^{\mathbb{N}}=\left(k e_{1}\right)^{\mathbb{N}} \oplus \cdots \oplus\left(k e_{d}\right)^{\mathbb{N}}
$$

and any $x \in V^{N}$ may be written $x=\alpha_{1} e_{1}+\cdots+\alpha_{d} e_{d}$ with $\alpha_{i} \in k^{\mathbb{N}}$. Accordingly, for $x \in V^{\mathbb{N}}$ we let

$$
\varphi(x)=\varphi\left(\alpha_{1}\right) e_{1}+\cdots+\varphi\left(\alpha_{d}\right) e_{d} .
$$

It is easy to see for any $v \in V$ and $\alpha \in k^{\mathbb{N}}$ that $\varphi(\alpha v)=\varphi(\alpha) v$. In particular, $\varphi: V^{\mathbb{N}} \rightarrow V$ depends only on the given $k$-linear map $\varphi: k^{\mathbb{N}} \rightarrow k$.

If $V$ is of infinite dimension, let $L \subseteq V^{\mathbb{N}}$ be the (directed) union of $U^{\mathbb{N}}$ over the finite dimensional subspaces $U$ of $V$. Then $k^{\bar{N}} \oplus L \subseteq k^{\mathbb{N}} \oplus V^{\mathbb{N}}$ is an $R$-invariant submodule, and, by the observations above, the $k$-linear map $k^{\mathbb{N}} \rightarrow k$ has a unique extension to an $R$-linear map $\varphi: k^{\mathbb{N}} \oplus L \rightarrow R$. Clearly, a vector space complement $K$ to $L$ inside $V^{\mathbb{N}}$ is $R$-invariant. Hence, with the value zero on $K$, the map $\varphi$ extends to an $R$-linear map $R^{\mathbb{N}} \rightarrow R$.

With $V$ of countable dimension, we have an example of a ring of bounded type which is $\aleph_{0}$-compact but not Artinian.

Example 5.3. The ring of the previous example in the case of countable dimension may be given as the algebra $R=k\left[e_{0}, e_{1}, e_{2}, \ldots\right]$ ( $k$ a field) with the relations $e_{i} e_{j}=0$ for all $i, j$; in particular $e_{i}^{2}=0$ for all $i$. It is worthwhile to notice that if we keep the relations for $i \neq j$, but require that $e_{i}^{2} \neq 0$ for all $i$ then the resulting ring $R$ is almost slender, and, in particular, it is not $\aleph_{0}$-compact. Indeed, if $\varphi: R^{\mathbb{N}} \rightarrow R$ is any linear map, set $e:=\left(e_{0}, e_{1}, \ldots\right)$, and choose the sequence $\lambda \in R^{\mathbb{N}}$ as in Lemma 3.6 with $\varphi(\lambda e)=0$ and, say, $\lambda_{p}=1$. Then $0=e_{p} \varphi(\lambda e)=e_{p}^{2} \varphi_{p}$, and hence $\varphi_{p}$ is a zero divisor. With $e_{i}$ nilpotent for all $i$, for instance $e_{i}^{3}=0$, the ring $R$ is perfect, and hence nonslender, see Example 5.4.

Example 5.4. A perfect ring is nonslender. Indeed, the assertion is an immediate consequence of Lemma 2.1 since it follows from Definition 3.8 that a perfect ring has simple ideals.

Example 5.5. Product rings provide obvious examples of rings with zero divisors. For instance, the ring $R:=\mathbb{Z} \times \mathbb{Q}$ is almost slender, because the first factor is slender, but $R$ is nonslender, because the second factor is nonslender (in fact, $\aleph_{0}$-compact).

For general results, consider a product of (non-zero) rings $R=\prod_{i \in I} R_{i}$ over an arbitrary index set $I$. Clearly, maps $\varphi: R^{\mathbb{N}} \rightarrow R$ may be identified with families $\left(\varphi^{i}\right)$ of maps $\varphi^{i}: R_{i}^{\mathbb{N}} \rightarrow R_{i}$. Accordingly, the following equivalences hold: Let $\wp$ be any of the properties 
"not almost slender", "nearly compact", or " $\aleph_{0}$-compact". Then $R$ has property $\wp$ iff every $R_{i}$ has property $\wp$.

For the property "slender" the results are more subtle. If the index set $I$ is finite, then $R$ is slender iff each $R_{i}$ is slender. If $I$ is infinite, then the product ring $\prod_{i} R_{i}$ is never slender.

To prove the last assertion choose an embedding $\mathbb{N} \subseteq I$. For $j \in \mathbb{N}$ let $e_{j} \in R$ be the family whose $i$ 'th coordinate is 1 for $i=j$ and zero otherwise. Then, clearly, $\varphi(x):=\sum x_{j} e_{j}$ is a well defined map $\varphi: R^{\mathbb{N}} \rightarrow R$, and $\varphi_{n}=e_{n} \neq 0$. Hence $R$ is nonslender.

Example 5.6. If $R=k\left[x_{1}, \ldots, x_{n}\right]$ is finitely generated over a field $k$, and of Krull dimension $d \geq 1$ then $R$ is almost slender. Indeed, by Noether's Normalization Lemma, $R$ is finitely generated as module over a subring $R_{0}=k\left[t_{1}, \ldots, t_{d}\right]$ which is a polynomial ring in $d$ variables. The subring is slender by 2.7 , and so $R$ is almost slender by 2.4 .

This result is improved in Theorem 6.4.

Example 5.7. Let $R_{0} \subseteq R_{1} \subseteq R_{2} \subseteq \cdots$ be an increasing sequence of subrings and inclusions and let $R=\bigcup R_{i}$ be the union. Assume that there exist non-zero elements $t_{i} \in R_{i}, i \geq 1$, such that $t_{i} R \cap R_{i-1}=0$. Then $R$ is almost slender, as it follows from the Key Lemma 2.6(1).

For instance, over a field $k$, let $R_{0}:=k$ and define $R_{i}, i \geq 1$ as the power series ring $R_{i-1}\left[\left[T_{i}\right]\right]$. Then each $R_{i}$ is $\aleph_{0}$-compact, but the union $R$, the ring of "finitistic" power series in $X_{1}, X_{2}, \ldots$, is slender.

A second example is the union where $R_{i}$, depending on the parity of $i$, is equal to $R_{i-1}\left[T_{i}\right]$ or $=R_{i-1}\left[\left[T_{i}\right]\right]$.

Example 5.8. Consider the ring $R$ of formal Laurent series over a ring $R_{0}$, that is, $R:=$ $R_{0}[[X]]\left[X^{-1}\right]$. The ring $R$ is slender if there exists in $R_{0}$ a regular element $p$ such that $\bigcap p^{n} R_{0}=0$. This is the result in the Corollary in Note 2.10; in particular, $R$ is slender if $R_{0}$ is a Noetherian ring without simple ideals, see Lemma 6.7.

On the contrary, $R$ is nonslender if $R_{0}$ contains a simple ideal. Indeed, assume that $R_{0}$ contains a simple ideal, that is, an ideal $r R_{0}$ such that the annihilator $\mathfrak{m}_{0}:=\operatorname{Ann}_{R_{0}}(r)$ is a maximal ideal of $R_{0}$. As observed in Lemma 2.1, a ring containing a simple ideal is nonslender. Hence it suffices to prove that $R$ contains a simple ideal. But clearly, $\operatorname{Ann}_{R}(r)$ is the ideal $\mathfrak{m}:=\mathfrak{m}_{0}[[X]]\left[X^{-1}\right]$ of series with coefficients in $\mathfrak{m}_{0}$, and it is maximal since $R / \mathfrak{m}$ is the field $\left(R / \mathfrak{m}_{0}\right)[[X]]\left[X^{-1}\right]$.

Let us finally note that $R$ is also slender if $R_{0}$ slender. This assertion is a special case (obtained with $\tau(f):=$ constant term in $f$ ) of a more general result:

Assume that $R_{0}$ is a subring of a ring $R$ such that there exists an $R_{0}$-linear map $\tau: R \rightarrow R_{0}$ for which the pairing $R \otimes_{R_{0}} R \rightarrow R_{0}$ determined by $s \otimes t \mapsto \tau(s t)$ is non-degenerate. Then, if $R_{0}$ is slender then so is $R$.

Indeed, assume indirectly that there exists an $R$-linear map $\varphi: R^{\mathbb{N}} \rightarrow R$ such that $u_{n}:=\varphi\left(1_{n}\right)$ is non-zero for all $n$. Then, by hypothesis, there exists for every $n$ an element $v_{n} \in R$ with $\tau\left(u_{n} v_{n}\right) \neq 0$. Let $v \in R^{\mathbb{N}}$ be the sequence $v=\left(v_{n}\right)$. Then, for the $R_{0}$-linear map $\varphi_{0}: R_{0}^{\mathbb{N}} \rightarrow R_{0}$ defined as the composition,

$$
\varphi_{0}: R_{0}^{\mathbb{N}} \hookrightarrow R^{\mathbb{N}} \stackrel{v}{\rightarrow} R^{\mathbb{N}} \stackrel{\varphi}{\rightarrow} R \stackrel{\tau}{\rightarrow} R_{0},
$$

we have $\varphi_{0}\left(1_{n}\right)=\tau\left(u_{n} v_{n}\right) \neq 0$, contradicting that $R_{0}$ is slender by hypothesis. 
Example 5.9. Consider for a field $k$ the subring $R \subseteq k^{\mathbb{N}}$ of eventually constant sequences. It is a consequence of Lemma 4.13 that $R$ is almost slender. The result is also a consequence of Theorem 3.10: $R$ is not perfect since the idempotent ideals $1_{[n, \infty)} R$ form a strictly decreasing chain, and $R$ is countably generated over $k$, for instance by the idempotent sequences $\delta_{n}$, equal to 1 at the $n$ 'th coordinate and 0 elsewhere.

For a more general result, see Example 5.12.

Example 5.10. Let $R$ be a Noetherian domain. Then the implication $\left(\aleph_{0}-\mathrm{c}\right) \Rightarrow(\mathrm{nl}-\mathrm{c})$ is a biimplication, and these (equivalent) conditions hold iff $R$ is a local complete domain (see Theorem 6.3); in particular, $R$ is half slender iff $R$ is not a local complete domain.

The implication $\left(\aleph_{0}-\mathrm{c}\right) \Rightarrow$ (nl-c) is not reversible for general domains. Indeed, to construct a domain $S$ which is nearly compact, but not $\aleph_{0}$-compact, consider the power series ring $R:=\mathbb{Q}[[X]]$. Let $S \subseteq R$ be the subring of series whose constant term belongs to $\mathbb{Z}_{(p)}$, and let $S_{0}=\mathbb{Z}_{(p)}$ be the subring of constants in $S$. Note that $S_{0}$ is a countable domain and not a field, and hence slender.

The ring $R$ is a local complete Noetherian domain, and hence, by Theorem $6.3, \aleph_{0^{-}}$ compact. In particular, $R$ is nearly compact. Multiplication by $X$ defines an $S$-linear injection $R \rightarrow S$. As a consequence, $R$ is torsionless over $S$, and hence, by Proposition 2.4(b), $S$ is nearly compact.

However, $S$ is not $\aleph_{0}$-compact. Indeed, the maximal ideal $X R$ of $R$ is an ideal of $S$, and $S / X R=S_{0}$. Thus the inclusion $S_{0} \hookrightarrow S$ is split. So by a result similar to the results in 2.4 (more precisely, see [JJT, Proposition 2.1(c"), p. 897]), if $S$ were $\aleph_{0}$-compact, the subring $S_{0}$ would be $\aleph_{0}$-compact as well, a contradiction since $S_{0}$ was slender.

Note that the localization $S[1 / p]=\mathbb{Q}[[X]]$ is $\aleph_{0}$-compact. Hence, in addition, $S$ is an example of a (non-Noetherian) domain with a proper localization which is $\aleph_{0}$-compact, but not a field, cf. Proposition 2.9 and Example 5.6.

Example 5.11. Let $R$ be a reduced ring of bounded type, and not a finite product of fields. It follows from Theorem 3.10 that $R$ is almost slender. We show by an example that such a ring, even in the class of von Neumann regular rings, can be nonslender, and, with a more delicate example, it can be slender. For a nonslender example, let $k$ be a field, and let $R \subset k^{\mathbb{N}}$ be the subring of eventually constant sequences. Clearly, the ideal $1_{0} R$ is a simple ideal. Hence, as observed in Example 5.4, $R$ is nonslender,

To obtain a slender example we note that for $R$ to be slender, it suffices, by Proposition 3.11 , to show that $R$ has no simple ideal. For the example let $V$ be the full infinite binary rooted tree. So $V$ has a root, and every node has a pair of children as the immediate successors. The set $V_{n}$ of nodes of depth $n$ has $2^{n}$ elements; the set $V_{0}$ contains only the root. The map $V_{n+1} \rightarrow V_{n}$, mapping a node of positive depth to its parent, is a surjective 2-1-map, and the system of these maps defines a cosystem of finite dimensional $k$-algebras and injections,

$$
k^{V_{0}} \rightarrow k^{V_{1}} \rightarrow k^{V_{2}} \rightarrow \cdots
$$

Let $R$ be the colimit $R:=\lim _{\longrightarrow} k^{V_{n}}$. Clearly, $R$ is von Neumann regular of bounded type. For

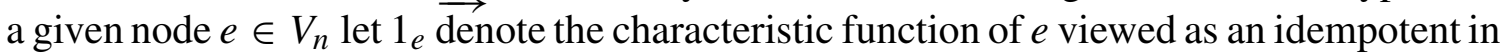
$k^{V_{n}}$. If $e^{\prime}$ and $e^{\prime \prime}$ denote, respectively, the left and right child of $e$, then under the inclusion $k^{V_{n}} \rightarrow k^{V_{n+1}}$ we have $1_{e}=1_{e^{\prime}}+1_{e^{\prime \prime}}$ and $1_{e^{\prime}} 1_{e^{\prime \prime}}=0$. Consider a non-zero element of $R$, represented by a non-zero vector $x \in k^{V_{n}}$. Let $e \in V_{n}$ be a node such that the coordinate $x_{e}$ is non-zero. Then, clearly, we have $1_{e} \in x R$. Moreover, since $1_{e^{\prime}} 1_{e^{\prime \prime}}=0$ we have $1_{e^{\prime}}=1_{e} 1_{e^{\prime}}$, and hence $1_{e^{\prime}} \in 1_{e} R$; in fact it is a strict inclusion $1_{e^{\prime}} R \subset 1_{e} R$ since $1_{e^{\prime \prime}} \notin 1_{e^{\prime}} R$. Thus $x R \supseteq 1_{e} R \supset 1_{e^{\prime}} R$. Therefore, the ideal $x R$ is not simple. 
Note in passing that the limit $P:=\lim V_{n}$ is the set of infinite paths from the root, and that the natural map is an embedding $R \subset k^{P}$.

Example 5.12. The product ring $k^{\mathbb{N}}$, where $k$ is a field, is $\aleph_{0}$-compact, see for instance Example 5.5. Let $R \subset k^{\mathbb{N}}$ be a proper subring containing $k^{(\mathbb{N})}$. Then $R$ is almost slender but not slender.

Indeed, denote by $\delta_{n} \in R \subseteq k^{\mathbb{N}}$ the sequence whose only non-zero coordinate is the $n$ 'th, equal to $1 \in k$. Clearly each $\delta_{n}$ generates a simple ideal of $R$. In particular, $R$ is nonslender by Example 5.4.

To prove that $R$ is almost slender we assume, indirectly, that there exists an $R$-linear map $\varphi: R^{\mathbb{N}} \rightarrow R$ such that each $\varphi_{n}$ is regular in $R$. Denote by $r_{n} \in k$ the $n$ 'th coordinate of $\varphi_{n}$. It is given by the equation $r_{n} \delta_{n}=\varphi_{n} \delta_{n}$. As $\varphi_{n}$ is regular in $R$ and $R$ contains $\delta_{n}$ it follows that $r_{n} \neq 0$. In particular, $r_{n}$ is invertible in $k$.

Let $a \in k^{\mathbb{N}}$ be any sequence, and consider the value

$$
h:=\varphi\left(a_{0} r_{0}^{-1} \delta_{0}, a_{1} r_{1}^{-1} \delta_{1}, \ldots\right) .
$$

The elements $\delta_{i}$ are orthogonal idempotents of $R$. Hence multiplication by $\delta_{n}$ yields the equation,

$$
h \delta_{n}=\varphi\left(0, \ldots, 0, a_{n} r_{n}^{-1} \delta_{n}, 0, \ldots\right)=a_{n} r_{n}^{-1} \varphi_{n} \delta_{n}=a_{n} \delta_{n} .
$$

The equations $h \delta_{n}=a_{n} \delta_{n}$ for all $n$ show that $h=\left(a_{0}, a_{1}, \ldots\right)$. As $h \in R$, this is a contradiction when the sequence $a$ is chosen outside $R$.

Example 5.13. The result in the previous example has a powerful extension:

Let $T$ be a set, and consider the k-algebra $k^{T}$ of functions $f: T \rightarrow k$. Let $U_{0}, U_{1}, \ldots$ be a sequence of pairwise disjoint subsets of $T$ and let $\delta_{0}, \delta_{1}, \delta_{2}, \ldots$ be a sequence of non-zero functions in $k^{T}$ such that $\operatorname{Supp}\left(\delta_{n}\right) \subseteq U_{n}$ for all $n$. Finally, let $R \subseteq k^{T}$ be a subalgebra. Assume that $R$ contains all the functions $\delta_{n}$, and assume for every sequence $t \in \prod_{n} U_{n}$ (that is, $t_{n} \in U_{n}$ for all $n$ ) that the evaluation map $\operatorname{Ev}_{t}: R \rightarrow k^{\mathbb{N}}$, given by $f \mapsto\left(f\left(t_{n}\right)\right.$ ), is not surjective. Then $R$ is almost slender.

The argument is essentially as in the previous example: Assume for $\varphi: R^{N} \rightarrow R$ that $\varphi_{n}$ is regular in $R$. As $\delta_{n} \neq 0$ it follows that $\varphi_{n} \delta_{n} \neq 0$, that is, there exists an element $t_{n}$ such the $\left(\varphi_{n} \delta_{n}\right)\left(t_{n}\right) \neq 0$. After a multiplication of $\delta_{n}$ by a constant, we may assume that $\left(\varphi_{n} \delta_{n}\right)\left(t_{n}\right)=1$. As $\delta_{n}\left(t_{n}\right) \neq 0$ we have $t_{n} \in U_{n}$.

Let $a=\left(a_{0}, a_{1}, \ldots\right)$ be any sequence in $k^{\mathbb{N}}$, and consider the function,

$$
h:=\varphi\left(a_{0} \delta_{0}, a_{1} \delta_{1}, \ldots\right)
$$

Multiply by $\delta_{n}$. As the functions $\delta_{n}$ are orthogonal, it follows that

$$
h \delta_{n}=\varphi\left(0, \ldots, 0, a_{n} \delta_{n}^{2}, 0, \ldots\right)=a_{n} \varphi_{n} \delta_{n}^{2} .
$$

Evaluate at $t_{n}$ and divide by $\delta_{n}\left(t_{n}\right)$ to obtain

$$
h\left(t_{n}\right)=a_{n} \varphi_{n}\left(t_{n}\right) \delta_{n}\left(t_{n}\right)=a_{n} .
$$

Hence, under the evaluation map, we have $\operatorname{Ev}_{t}(h)=a$, a contradiction when $a$ is chosen outside the image of the evaluation map.

Clearly, the expression $\sum r_{n} \delta_{n}$ for $r \in R^{\mathbb{N}}$ defines an $R$-linear map $R^{\mathbb{N}} \rightarrow k^{T}$, and $R$ is nonslender it this map has image contained in $R$. This observation may be applied to some of the rings in the next example. 
Example 5.14. Let $T$ be a "nice" topological space, say a "Tychonoff space" (i.e., infinite, Hausdorff, and closed subset may be separated from a point in the complement by a continuous real function). It follows from the Hausdorff property that there exists a sequence $U_{0}, U_{1}, \ldots$ of non-empty open pairwise disjoint subsets $U_{n} \subseteq T$. By the separation property there exists for each $n$ a non-zero continuous function $\delta_{n}$ with $\operatorname{Supp}\left(\delta_{n}\right) \subseteq U_{n}$. Hence it follows from the previous result that any subalgebra of $\mathbb{R}^{T}$ containing the functions $\delta_{n}$ and having the "evaluation property" is almost slender.

For instance, the algebra of all bounded functions $f: T \rightarrow \mathbb{R}$ is almost slender. If $T$ is compact, then the algebra of all continuous functions $T \rightarrow \mathbb{R}$ is almost slender (and nonslender).

Assume in addition that there exists in $T$ a non-isolated point $u$ having a countable basis for its open neighborhoods. Then the sequence of open sets $U_{n}$ as above may be chosen in such a way the any point sequence $t \in \prod U_{n}$ converges to $u$. Hence, again by the result in Example 5.13, if $R \subseteq \mathbb{R}^{T}$ is any subalgebra containing the $\delta_{n}$ and consisting of functions that are continuous at $u$, then $R$ is almost slender. For instance, the algebra of all continuous real functions on $T$ is almost slender (and nonslender).

Note that the results may be applied to rings of real sequences: The ring of all bounded sequences and the ring of all convergent sequences are almost slender.

\section{Noetherian rings}

Lemma 6.1. Assume that $R$ is Noetherian, and let $\mathfrak{p} \subseteq R$ be a minimal prime. If the residue ring $R / \mathfrak{p}$ is slender, then $R$ is almost slender. Conversely, if $R$ is slender, then $R / \mathfrak{p}$ is slender.

Proof. In the proof we use that a minimal prime $\mathfrak{p}$ is an associated prime, that is, $\mathfrak{p}=\operatorname{Ann}(a)$ is the annihilator of an element $a \in R$. In fact, the lemma and the following proof hold for any associated prime.

The proof of the first assertion is indirect. Assume that $R$ is not almost slender, that is, assume there exists an $R$-linear map $\varphi: R^{\mathbb{N}} \rightarrow R$ such that $a_{n}=\varphi_{n}$ is regular for all $n$. By Lemma 2.12, $\varphi$ induces a linear map $\bar{\varphi}:(R / \mathfrak{p})^{\mathbb{N}} \rightarrow R / \mathfrak{p}$. Since $\mathfrak{p}$ is an associated prime, it contains no regular elements. Hence $\bar{\varphi}_{n}=\left(a_{n} \bmod \mathfrak{p}\right)$ is non-zero for all $n$, a contradiction since $R / \mathfrak{p}$ is slender.

Conversely, assume that $\bar{R}:=R / \mathfrak{p}$ is nonslender, that is, assume there exists an $\bar{R}$-linear map $\bar{\varphi}:(R / \mathfrak{p})^{\mathbb{N}} \rightarrow \bar{R}$ such the $\bar{\varphi}_{n} \neq 0$ for all $n$. Since $\mathfrak{p}=\operatorname{Ann}(a)$ the $R$-linear map $r \rightarrow r a$ induces an $R$-linear injection $\bar{R} \hookrightarrow R$. So the natural composition,

$$
\varphi: R^{\mathbb{N}} \rightarrow(R / \mathfrak{p})^{\mathbb{N}} \stackrel{\bar{\varphi}}{\rightarrow} R / \mathfrak{p} \hookrightarrow R,
$$

has $\varphi_{n} \neq 0$ for all $n$.

Lemma 6.2. Assume that $R$ is Noetherian and nearly compact. If $R$ is an integral domain then $R$ is local. In general, $R$ is semilocal.

Proof. The case of an integral domain is Proposition [JJT, Lemma 4.1, p.901]. Consider the general case. Let $\mathfrak{p}$ be a minimal prime of $R$. Then, as in the proof of the previous lemma, the residue ring $R / \mathfrak{p}$ is nearly compact since $R$ is nearly compact. Hence, by the integral case, $R / \mathfrak{p}$ is local. In other words, there is only one maximal ideal containing $\mathfrak{p}$. Consequently, since there are only finitely many minimal prime ideals, there are only finitely many maximal ideals. 
Theorem 6.3. For a Noetherian ring $R$ the following four conditions are equivalent:

(i) $R$ is a finite product of local complete rings,

(ii) $R$ is algebraically compact,

(iii) $R$ is $\aleph_{0}$-compact,

(iv) $R$ is nearly compact.

In particular, an Artinian ring is $\aleph_{0}$-compact.

Moreover, if the conditions hold, then $R$ is complete with respect to any non-discrete Hausdorff filtration.

Proof. For a local Noetherian ring with maximal ideal $\mathfrak{m}$ completeness without further qualification refers to the $\mathfrak{m}$-adic filtration. It is Hausdorff (by Krull's Intersection Theorem), and it is discrete only for Artinian rings.

The equivalence (i) $\Leftrightarrow$ (ii) is proved in [J-L, Theorem 11.3, p. 283]. The implications (ii) $\Rightarrow$ (iii) and (iii) $\Rightarrow$ (iv) follow from the definitions. Finally, to prove the equivalence of the four conditions we prove the implication (iv) $\Rightarrow$ (i). As a consequence we then have a proof of the implication (iii) $\Rightarrow$ (ii) stated in [G-J, Theorème 9.1, p. 282].

Assume (iv). It is well known that (i) holds iff $R$ is semilocal and complete with respect to the $\mathfrak{r}$-adic filtration $\left(\mathfrak{r}^{n}\right)$, where $\mathfrak{r}$ is the Jacobson radical of $R$. We verify the latter property. By Lemma $6.2, R$ is semilocal, so it remains to verify that $R$ is complete. The proof in the case of an integral domain is given in [JJT, Theorem 4.2, p. 901]. We give here the more involved proof for the general case.

Since $R$ is nearly compact there is, by Proposition 2.11(2), a regular element $u \in R$ such that $R$ is complete with respect to a filtration $\left(\mathfrak{a}_{n}\right)$ such that

$$
u \mathfrak{r}^{n} \subseteq \mathfrak{a}_{n} \subseteq \mathfrak{r}^{n}
$$

Let $\widehat{R}:=\lim R / \mathfrak{r}^{n}$ be the $\mathfrak{r}$-adic completion. As $R$ is complete with respect to the filtration $\left(\mathfrak{a}_{n}\right)$, we have $R=\lim R / \mathfrak{a}_{n}$, and the inclusion $R \hookrightarrow \widehat{R}$ is the limit of the surjective map of $\mathbb{N}$-systems $R / \mathfrak{a}_{n} \rightarrow R / \mathfrak{r}^{n}$. It follows from the inclusions $u \mathfrak{r}^{n} \subseteq \mathfrak{a}_{n}$ that multiplication by $u$ in $\widehat{R}$ factors through $R$. In other words, $u \widehat{R} \subseteq R$.

As is well known, $\widehat{R}$ is $R$-flat [Bo, Theoreme 3 , III.3.4, p. 68]. In particular, $u$ is not a zero divisor on $\widehat{R}$. Therefore, the multiplication $u: \widehat{R} \rightarrow R$ is injective. Consequently, as an $R$-module, $\widehat{R}$ is isomorphic to an ideal of $R$. In particular, $\widehat{R}$ is finitely generated over $R$. Therefore, by a result of Frankild and Sather-Wagstaff [F-SW, Theorem B, p. 2304], $R$ is $\mathfrak{r}$-adically complete. Hence the equivalence of the conditions has been proved.

An Artinian ring is a finite product of local Artinian rings, and here each factor is discrete and hence complete. Thus an Artinian ring satisfies (i). Therefore, as a consequence of (i) $\Rightarrow$ (iii), an Artinian ring is $\aleph_{0}$-compact.

The final assertion is a consequence of (iii) and Proposition 2.11(1).

Theorem 6.4. Assume that $R$ is a localization of a finitely generated $k$-algebra $R_{0}=$ $k\left[x_{1}, \ldots, x_{r}\right]$ ( $k$ a field). Then $R$ is either Artinian or almost slender.

Proof. Assume that $R$ is not Artinian. We have to prove that $R$ is almost slender. The proof in the case where $R_{0}$ is an integral domain is given in [JJT, Corollary 4.7, p. 902]. The general case is reduced to the integral case as follows.

As $R$ is not Artinian, there exists prime ideal $\mathfrak{p} \subset R$ which is minimal prime and not a maximal ideal. Then the residue ring $R / \mathfrak{p}$ is not a field. Moreover, if $\mathfrak{p}_{0}=\mathfrak{p} \cap R_{0}$ is the contraction, then $R_{0} / \mathfrak{p}_{0}$ is a finitely generated integral domain, and, by standard results on localization, $\mathfrak{p}=\mathfrak{p}_{0} R$ and $R / \mathfrak{p}$ is a localization of $R_{0} / \mathfrak{p}_{0}$. Hence, by the integral case, $R / \mathfrak{p}$ is slender. Therefore, by Lemma 6.1, $R$ is almost slender. 
Lemma 6.5. Let $R$ be a Noetherian 1-dimensional integral domain, and assume that $R$ is nonslender. Then $R$ is local.

Proof. This is [JJT, Lemma 5.3, p. 903], recalled for the convenience of the reader.

Theorem 6.6. Let $R$ be a Noetherian ring of dimension 1 . Then $R$ is not almost slender if and only if the equivalent conditions of Theorem 6.3 hold.

In particular, $R$ is almost slender if and only if $R$ is not semilocal and complete. Moreover, if $R$ is an integral domain then $R$ is slender if and only if $R$ is not local and complete.

Proof. If the equivalent conditions hold then, in particular, $R$ is $\aleph_{0}$-compact, and hence not almost slender.

Assume conversely that $R$ is not almost slender. Let $\mathfrak{p}$ be a minimal prime of $R$. If $\mathfrak{p}$ is not a maximal ideal, then $\operatorname{dim} R / \mathfrak{p}=1$, and $R / \mathfrak{p}$ is nonslender by Lemma 6.1 ; hence $R / \mathfrak{p}$ is local by the previous lemma. If $\mathfrak{p}$ is a maximal ideal, then $R / \mathfrak{p}$ is trivially local. Thus $R / \mathfrak{p}$ is local for any minimal prime $\mathfrak{p}$. As there are only finitely many minimal primes, there are the same finite number of maximal ideals. Hence $R$ is semilocal.

Let $\mathfrak{r}$ be the Jacobson radical. Then $\bigcap \mathfrak{r}^{n}=0$ by Krull's Intersection Theorem, and $\mathfrak{r}^{n} \neq 0$ for all $n$ since $R$ is not Artinian. Therefore, by Proposition 2.11(3), $R$ is complete with respect to a filtration the form

$$
R \supseteq p_{1} \mathfrak{r} \supseteq p_{2} \mathfrak{r}^{2} \supseteq \cdots
$$

with regular elements $p_{n}$. Consider a prime ideal $\mathfrak{p}$ containing $p_{n} \mathfrak{r}^{n}$. Either $\mathfrak{p}$ contains $p_{n}$; since $p_{n}$ is a regular element and $\operatorname{dim} R=1$ it follows that $\mathfrak{p}$ is a maximal ideal. Or, $\mathfrak{p}$ contains $\mathfrak{r}$, in which case $\mathfrak{p}$ is also a maximal ideal. Therefore, the residue ring $R / p_{n} \mathfrak{r}^{n}$ is Artinian, and, consequently, some power $\mathfrak{r}^{N}$ is contained in $p_{n} \mathfrak{r}^{n}$. In other words, the topology defined by $(\dagger)$ is equal to the $\mathfrak{r}$-adic topology. Thus $R$ is complete in this topology, and condition (i) of Theorem 6.3 has been verified.

Lemma 6.7. Let $R$ be a Noetherian ring without simple ideals. Then there exists a regular element $t \in R$ such that $\bigcap t^{n} R=0$.

Proof. Let $\mathfrak{p}_{1}, \ldots, \mathfrak{p}_{r}$ be the associated prime ideals of $R$. Then the union of the $\mathfrak{p}_{i}$ is the set of zero divisors. Choose for every $j$ a maximal ideal $\mathfrak{m}_{j} \supseteq \mathfrak{p}_{j}$. By hypothesis, no $\mathfrak{p}_{i}$ is maximal. Hence, by prime avoidance, $\mathfrak{m}_{j}$ is not contained in the union of the $\mathfrak{p}_{i}$. Accordingly, we may choose a regular element $t_{j} \in \mathfrak{m}_{j}$. Let $t$ be the product of the $t_{j}$. Then $t$ is a regular element contained in the intersection $\bigcap m_{i}$. We claim that $\bigcap t^{n} R=0$.

Let $S \subseteq R$ be the complement of the union $\mathfrak{m}_{1} \cup \cdots \cup \mathfrak{m}_{r}$, and consider the localization $S^{-1} R$. By the choice of the $\mathfrak{m}_{i}$, the set $S$ consists of regular elements. Hence the canonical map $R \rightarrow S^{-1} R$ is injective. As is well known, the ring $S^{-1} R$ is semi-local, with the maximal ideals $S^{-1} \mathfrak{m}_{1}, \ldots, S^{-1} \mathfrak{m}_{r}$. Their intersection is the Jacobson radical,

$$
\mathfrak{r}=S^{-1} \mathfrak{m}_{1} \cap \cdots \cap S^{-1} \mathfrak{m}_{r}
$$

and, by choice, $t \in \mathfrak{r}$. By Krull's Intersection Theorem, we have $\bigcap \mathfrak{r}^{n}=0$. Consequently $\bigcap t^{n} R=0$.

Theorem 6.8 (see El Bashir and Barka [BK-2, 4.4 Corollary, p. 2590]). Let $R$ be a Noetherian ring without simple ideals. If $R$ is nonslender, then $R$ is complete with respect to some non-discrete Hausdorff filtration: $R \supseteq \mathfrak{a}_{1} \supseteq \mathfrak{a}_{2} \supseteq \cdots$, with $\mathfrak{a}_{n} \neq 0$ and $\bigcap \mathfrak{a}_{n}=0$. 
Proof. Assume that $R$ is nonslender so that there exists an $R$-linear map $\psi: R^{\mathbb{N}} \rightarrow R$ with $\psi_{n} \neq 0$ for all $n$. By Lemma 6.7 we may choose a regular element $t \in R$ with $\bigcap t^{n} R=0$. Define $\varphi: R^{\mathbb{N}} \rightarrow R$ by the equation, for $x \in R^{\mathbb{N}}$,

$$
\varphi\left(x_{0}, x_{1}, x_{2}, \ldots\right)=\psi\left(x_{0}, t x_{1}, t^{2} x_{2}, \ldots\right) .
$$

Then $\varphi_{n}=t^{n} \psi_{n} \neq 0$ for all $n$. Hence the image ideal $\mathfrak{a}_{n}:=\varphi\left(R^{[n, \infty)}\right)$ is non-zero. Moreover, for $x \in R^{[n, \infty)}$ all the coordinates on the right side of (6.8.1) are divisible by $t^{n}$. Hence $\varphi\left(R^{[n, \infty)}\right) \subseteq t^{n} R$. Consequently, $\bigcap \mathfrak{a}_{n}=0$. Therefore, by the result of Dimitrić, see Remark 2.3, $R$ is complete with respect to filtration $\left(\mathfrak{a}_{n}\right)$.

Remark 6.9. The result in Theorem 6.8 is due to El Bashir and Barka; it is presented here with an alternative proof. Corollary 6.11 below for the reduced Noetherian case contains the extra information that the ideals of the filtration may be taken to be principal ideals. We have not been able to verify such a possibility in the general Noetherian case.

Lemma 6.10. Let $R$ be a Noetherian ring without simple ideals. Assume that there exists an $R$-linear map $\varphi: R^{\mathbb{N}} \rightarrow R$ such that no $\varphi_{n}$ is nilpotent. Then $R$ is complete with respect to a filtration with non-zero principal ideals $R \supseteq a_{1} R \supseteq a_{2} R \supseteq \cdots$ such that $\bigcap a_{n} R=0$.

Proof. The nilradical $\mathfrak{n}=\mathfrak{n}(R)$ is the intersection of the finitely many minimal prime ideals. Hence, for some minimal prime $\mathfrak{p}$ we have that $\varphi_{n} \notin \mathfrak{p}$ for infinitely many $n$. Restricting $\varphi$ if necessary, we may assume that $\varphi_{n} \notin \mathfrak{p}$ for all $n$.

By Lemma 6.7, there exists a regular element $t \in R$ such that $\bigcap t^{n} R=0$. Define a modification $\psi$ by the formula, for $x=\left(x_{0}, x_{1}, x_{2}, \ldots\right)$,

$$
\psi(x):=\varphi\left(q_{0} x_{0}, q_{1} x_{1}, q_{2} x_{2}, \ldots\right), \quad \text { where } q_{n}:=t^{n} \varphi_{0} \cdots \varphi_{n-1}\left(q_{0}=1\right) .
$$

Note that $\psi_{n}=q_{n} \varphi_{n}=t^{n} \varphi_{0} \cdots \varphi_{n}$ is non-zero because $\mathfrak{p}$ is a prime ideal, and $q_{n+1}=t \psi_{n}$. Clearly,

$$
\psi\left(R^{[n, \infty)}\right)=\psi_{n} R+\psi\left(R^{[n+1, \infty)}\right) .
$$

On the right side of (6.10.1), the second ideal is contained in $q_{n+1} R$, and $q_{n+1}=t \psi_{n}$. So the second ideal is contained in the first, and we conclude the $\psi\left(R^{[n, \infty)}\right)=\psi_{n} R$. As $\psi_{n} \in t^{n} R$, it follows that $\bigcap \psi\left(R^{[n, \infty)}\right)=0$. Therefore, with $a_{n}:=\psi_{n}$, it follows from the result of Dimitrić, see Remark 2.3, that $R$ is complete with respect to the filtration $\left(a_{n} R\right)$.

Corollary 6.11. A reduced Noetherian ring $R$ without simple ideals is slender iff $R$ is not complete with respect to any filtration of non-zero principal ideals $R \supseteq a_{1} R \supseteq a_{2} R \supseteq \cdots$ such that $\bigcap a_{n} R=0$.

\section{Augmenting the product algebra}

7.1. For an $\aleph_{0}$-compact ring $R$ the characterization ( $\aleph_{0}$-c') in Definition 1.2 yields an $R$-linear map $R^{\mathbb{N}} \rightarrow R$ vanishing on $R^{(\mathbb{N})}$, and mapping $1_{\mathbb{N}} \in R^{\mathbb{N}}$ to $1 \in R$. The source $R^{\mathbb{N}}$, as a product of copies of $R$, is an $R$-algebra with $1_{\mathbb{N}}$ as the unity, and $R^{(\mathbb{N})}$ is an ideal. It is tempting to ask if there exists such an $R$-linear map which in addition is a map of rings, or, equivalently, if there exists an augmentation on the residue algebra $R^{\mathbb{N}} / R^{(\mathbb{N})}$.

Theorem 7.2. For a Noetherian ring $R$ the following conditions are equivalent:

(i) There exists a map of $R$-algebras $\varphi: R^{\mathbb{N}} \rightarrow R$ vanishing on $R^{(\mathbb{N})}$.

(ii) $R$ is a finite product of local, complete rings with finite residue fields.

(iii) $R$ is nearly compact and the residue ring of $R$ modulo the Jacobson radical is a finite ring. 
Proof. The equivalence of (ii) and (iii) is immediate from Theorem 6.3.

If $\varphi: R^{\mathbb{N}} \rightarrow R$ is a map as in (i) then $R$ is $\aleph_{0}$-compact, and hence $R$ is a finite product of local complete rings. Accordingly, $\varphi$ is the finite product of algebra homomorphisms corresponding to the factors of $R$. Therefore, to prove the equivalence of (i) and (ii) we may assume that $R$ is local. Let $\mathfrak{m}$ be the maximal ideal of $R$.

Assume first (i). Since $\mathfrak{m}$ is finitely generated we have $\mathfrak{m} R^{\mathbb{N}}=\mathfrak{m}^{\mathbb{N}}$. Hence reduction modulo $\mathfrak{m}$ of $\varphi$ is a map of $R / \mathfrak{m}$-algebras $\bar{\varphi}:(R / \mathfrak{m})^{\mathbb{N}} \rightarrow R / \mathfrak{m}$ vanishing on $(R / \mathfrak{m})^{(\mathbb{N})}$. In particular, the kernel of $\bar{\varphi}$ is a maximal ideal in $(R / \mathfrak{m})^{\mathbb{N}}$.

Now, as is well known, if $k$ is any commutative ring and $\mathcal{F}$ is a filter on $\mathbb{N}$ there is an associated ideal $\mathfrak{z}(k, \mathcal{F}) \subset k^{\mathbb{N}}$ : a sequence $x \in k^{\mathbb{N}}$ belongs to $\mathfrak{z}(k, \mathcal{F})$ iff its zero set $\left\{n \mid x_{n}=0\right\}$ belongs to $\mathcal{F}$. If $\mathcal{F}$ is an ultrafilter then the ideal $\mathfrak{z}(k, \mathcal{F})$ contains $k^{(\mathbb{N})}$ iff $\mathcal{F}$ is non-principal. Moreover, for a non-principal ultrafilter $\mathcal{F}$ the composition $k \rightarrow k^{\mathbb{N}} \rightarrow k^{\mathbb{N}} / \mathfrak{z}(k, \mathcal{F})$ is an isomorphism if and only if the ring $k$ is finite. Indeed, if $k$ is finite, then for any sequence $x \in k^{\mathbb{N}}$ the set $\mathbb{N}$ is the disjoint union of the finitely many subsets $\left\{n \mid x_{n}=a\right\}$ for $a \in k$; hence for exactly one $a$ the corresponding subset belongs to $\mathcal{F}$, and modulo $\mathfrak{z}(k, \mathcal{F})$ the residue class of $x$ equals the residue class of the constant sequence $a$. Conversely, if $k$ is infinite there is a sequence $x$ with $x_{n} \neq x_{m}$ for $n \neq m$, and then the residue of $x$ modulo $\mathfrak{z}(k, \mathcal{F})$ is not equal to the residue of a constant sequence.

If $k$ is a field then the maximal ideals of $k^{\mathbb{N}}$ are exactly the ideals of the form $\mathfrak{z}(k, \mathcal{F})$ associated to ultrafilters $\mathcal{F}$.

Applied with $k:=R / \mathfrak{m}$ to the kernel of $\bar{\varphi}:(R / \mathfrak{m})^{\mathbb{N}} \rightarrow R / \mathfrak{m}$, it follows that $R / \mathfrak{m}$ is finite. Thus (ii) holds.

Conversely, assume Condition (ii). Fix a non-principal ultrafilter $\mathcal{F}$ on $\mathbb{N}$. Consider for $n \geq 1$ the ideal $\mathfrak{z}_{n}=\mathfrak{z}\left(R / \mathfrak{m}^{n}, \mathcal{F}\right)$. The ring $R / \mathfrak{m}^{n}$ is finite, since $R / \mathfrak{m}$ is finite. Hence the composition $R / \mathfrak{m}^{n} \rightarrow\left(R / \mathfrak{m}^{n}\right)^{\mathbb{N}} \rightarrow\left(R / \mathfrak{m}^{n}\right)^{\mathbb{N}} / \mathfrak{z}_{n}$ is an isomorphism. Thus we obtain an exact sequence,

$$
0 \rightarrow \mathfrak{z}_{n} \rightarrow\left(R / \mathfrak{m}^{n}\right)^{\mathbb{N}} \rightarrow R / \mathfrak{m}^{n} \rightarrow 0
$$

Consider the surjection $R / \mathfrak{m}^{n} \rightarrow R / \mathfrak{m}^{l}$ for $n \geq l$. Clearly, under the induced map $\left(R / \mathfrak{m}^{n}\right)^{\mathbb{N}} \rightarrow\left(R / \mathfrak{m}^{l}\right)^{\mathbb{N}}$, the zero set of a sequence $x \in\left(R / \mathfrak{m}^{n}\right)^{\mathbb{N}}$ contains the zero set of the image of $x$. Hence, under the induced map $\mathfrak{z}_{n}$ is mapped into $\mathfrak{z}_{l}$. Thus the entries in $\left(^{*}\right)$ form a short exact sequence of inverse systems.

Pass to the limits. As $R$ is complete we have $\lim _{\longleftarrow} R / \mathfrak{m}^{n}=R$, and, as a consequence, $\underset{\lim }{\longleftarrow}\left(\left(R / \mathfrak{m}^{n}\right)^{\mathbb{N}}\right)=R^{\mathbb{N}}$. So the result is an exact sequence,

$$
0 \rightarrow \underset{n}{\lim } \mathfrak{z}_{n} \rightarrow R^{\mathbb{N}} \rightarrow R .
$$

The limit of the $\mathfrak{z}_{n}$ is an ideal, and it contains $\mathfrak{z}(R, \mathcal{F})$; in particular, the kernel of $R^{\mathbb{N}} \rightarrow R$ contains $R^{(\mathbb{N})}$. The map $R^{\mathbb{N}} \rightarrow R$ is clearly a homomorphism of rings, and $R$-linear. Hence it satisfies the conditions in (i).

\section{REFERENCES}

[EB-K1] R. El Bashir and T. Kepka, On when small semiprime rings are slender, Comm. Algebra 24 (1996), 1575-1580.

[EB-K2] R. El Bashir and T. Kepka, On when commutative noetherian rings are slender, Comm. Algebra 25 (1997), 2585-2591.

[EB-K3] R. El Bashir and T. Kepka, Modules commuting (via Hom) with some limits, Fund. Math. 155 (1998), 271-292.

[Ba] H. Bass, Finitistic dimension and a homological generalization of semi-primary rings, Trans. Amer. Math. Soc. 90 (1960), 466-488. 
[Bo] N. Bourbaki, Algèbre commutative, chapitres III et IV, Élements de mathématique, Fasc XXVIII, Herman, Paris, 1967.

[D] R. Dimitrić, Slenderness in Abelian categories, in book: Abelian Group Theory, Proceedings, Honolulu 1982/83. Eds. R. Göbel, L. Lady, A. Mader. LNM 1006, Springer-Verlag, Berlin, 1983, pp. 375-383.

[E-M] P. C. Eklof and A. H. Mekler, Almost free modules, North-Holland Mathematical Library vol. 46, North-Holland, Amsterdam, 1990.

[F-SW] A. Frankild and S. Sather-Wagstaff, Detecting completeness from Ext-vanishing, Proc. Amer. Math. Soc. 136 (2008), 2303-2312.

[G] F. Q. Gouvêa, p-adic Numbers, An Introduction, Universitext, Springer-Verlag, Berlin, 1993.

[G-J] L. Gruson and C. U. Jensen, Dimensions cohomologiques relieés aux foncteurs $\varliminf^{(i)}$, Séminaire d'Algèbre Paul Dubreil et Marie-Paule Malliavin 1980, Lecture Notes in Mathematics Volume 867, Springer, 1981, pp. 234-294.

[J] N. Jacobson, Lectures in Abstract Algebra, vol. II, Linear Algebra, van Nostrand Company, 1953.

[JJT] C. U. Jensen, S. Jøndrup, and A. Thorup, Slender domains and compact domains, Forum Math. 29 (2017), 893-904.

[J-L] C. U. Jensen and H. Lenzing, Model-theoretic algebra with particular emphasis on fields, rings, modules, Algebra, Logic and Applications 2, Gordon and Breach Science Publishers, New York, 1989.

[ON] J. D. O’Neill, Slender modules over various rings, Indian J. pure appl. Math. 22 (1991), $287-293$.

[P] B. Poonen, Maximally complete fields, Enseign. Math. (2) 39 (1993), 87-106.

[R] F.P. Ramsey, On a problem of formal logic, Proc. London Math. Soc. Ser. 230 (1930), $264-285$.

Universitetsparken 5, DK-2100 Copenhagen, Denmark

E-mailaddress: cujensen@math.ku.dk, jondrup@math.ku.dk, thorup@math.ku.dk 